\title{
About the foundation of the Kubo Generalized Cumulants theory. A revisited and corrected approach
}

Marco Bianucci ${ }^{1, a)}$ and Mauro Bologna 2 ,a)

1) Istituto di Scienze Marine, Consiglio Nazionale delle Ricerche (ISMAR - CNR), 19032 Lerici $(S P)$, Italy

${ }^{2)}$ Instituto de Alta Investigación, Universidad de Tarapacá, Casilla 6-D Arica, Chile

(Dated: 22 November 2019)

More than fifty years ago, in a couple of seminal works 1,2 Kubo introduced the important idea of generalized cumulants, extending to stochastic operators this concept, implicitly introduced by Laplace in 1810. Kubo's idea has been applied in several branches of physics, where the result of the average process is a Lioville operator or an effective time evolution operator for the density matrix of spin systems or the reduced density matrix for boson-fermions etc. Despite this success, the theoretical developments in these Kubo works pose problems that were highlighted many years ago by Fox and van Kampen and never solved. These weaknesses and errors, in particular concerning the factorization property of exponentials of cumulants and the explicit expressions that give generalized cumulants in terms of generalized moments and vice-versa, caused some perplexity (and confusion) about the possible application of this procedure, limiting its use, in practice. In the present paper, we give a sound ground to the approach to cumulant operators, working in a general framework that shows the potentiality of the old Kubo's idea, today not yet fully exploited. It results that for the same moment operators, different definitions of generalized cumulants can be adopted. A general Kubo-Meeron closed-form formula giving cumulant operators in terms of moment operators cannot be obtained, but the reverse one, cumulants in terms of operators, is given and, noticeably, formally it does not depend on the specific nature of the moments, but just on the definition of the generalized cumulants.

\footnotetext{
a) marco.bianucci@cnr.it
} 


\section{INTRODUCTION}

Cumulants where implicitly introduced by Laplace in $1810^{\underline{3}-5}$ in his proof of the central limit theorem, as coefficients of the power expansion of the logarithm of the characteristic function. It is noticeable that nobody thought of defining the coefficients of this expansion as separate entities and to study their very important properties until Thiele in 1889. Cumulants have been recognized as a very powerful tool in any field of random processes: cumulants change in a very simple way when the underlying random variable is subject to an affine transformation, cumulants may be used in a simple way to describe the difference between a distribution and its simplest Gaussian approximation and, due to the factorization property of the exponential of a sum of commuting quantities, the cumulants have the useful fundamental property that they are not vanishing if and only if they refer to variables that are statistically "connected" to each other.

In the early 60 s of the past century, the great scientist R. Kubo had the idea to generalize the definition of cumulants 1.2 (K62-63 hereafter), including stochastic operators, as we shall see hereafter. At first sight, an extension of the cumulant concept to non-commuting stochastic processes comes up against the fact that for non-commuting quantities the factorization property of exponentials does not hold. However, Kubo proposed a way to overcome this problem by extending the definition of exponentials. After this old work by Kubo, which, as we shall see, lacked some formal gaps and contained some errors (giving rise to a series of critical remarks $\left.\frac{6,7}{7}\right)$, the theory of cumulants made much progress. In particular, we consider very interesting the recent formal framework where concepts related to combinatorial calculation, connected or crossed partitions etc. are used and the old results on the Möbius inversion on the lattice of partitions play a crucial role (e.g.,,$\left.\frac{8,9}{9}\right)$. However, in this approach, references to the notion of probability, average, or characteristic function do not enter, thus physics, or, more in general, the nature (and the definition) of the generalized moment generating function is a starting point, rather than a result. On the other hand, once the moment generating function is given and our formula that gives moments in terms of cumulants is obtained (see Eq. (63) ), we believe that the theory of lattice of partitions could be a powerful tool to obtain the inverse formula (cumulants in terms of moments) for some specific case of interest, a result that, as we shall show, cannot be achieved by our general approach. 
In this work, we shall focus on the classical Kubo's method where (generalized) moments arise by problems in classical o quantum physics (and beyond physics, too) and the (generalized) standard cumulants technique are introduced as a tool for some systematic expansion of these moment-operators of interest. In these problems, the Kubo's original idea remains the pillar on which to base any extension of the concept of cumulants and it is almost always referred (and cited) as the basis of any papers (also the more recent ones) concerning, e.g., the reduced or stochastic Liouville equation (see below), the evolution of density matrix of spin systems 10,11 or the reduced density matrix for many body boson or fermion systems (e.g., $\stackrel{12-19}{ }$ ). However, although mentioned, the general results of K62-63 are not used, and a more analytical approach, case dependent, is adopted. A fairly recent example is the case of the above-mentioned works treating problems in the field of many-electron densities and reduced density matrices and related Green functions. The analytical developments in these works mainly aim at obtaining a direct expression that gives reduced density matrices (or Green functions) in terms of generalized cumulants ( $\sec ^{\underline{15}}$ (TABLE II) or $\stackrel{14}{ }$ ), but, as it we shall show in this work, this formula can be given in a much more general form (see Lemma 4 , Eq. (64)), formally independent of the specific nature of the generalized moments (in this case density matrices, Green functions etc..). This example will be extensively discussed in Section IV D 1.

The reason why, although so much cited, Kubo's work is not being fully exploited is precisely that, as some authoritative remarks have pointed out, some steps and conclusions are not well justified, and some results are actually wrong. To remedy this situation we shall review shortly the Kubo's idea of generalized cumulants by using a specific example borrowed from classical statistical physics.

\section{THE KUBO'S IDEA FOR GENERALIZED CUMULANTS AND THE RELATED CRITICISM}

Let us assume to have a (classical or quantum) density function (DF) $P_{\xi}(\boldsymbol{x} ; t)$ where $\boldsymbol{x}$ are variables of interest or observables that we can measure in some way, while $\xi$ represents a collection of variables that we cannot measure and of which we just know a DF $\rho(\xi)$ (or a probability measure $\mathrm{d} \mu:=\rho(\xi) \mathrm{d} \xi)$ over which we can average. We assume that $P_{\xi}(\boldsymbol{x} ; t)$ 
satisfies the following generic equation of motion:

$$
\partial_{t} P_{\xi}(\boldsymbol{x} ; t)=\mathcal{L}_{0}(\boldsymbol{x}) P_{\xi}(\boldsymbol{x} ; t)+\mathcal{L}_{I}(\boldsymbol{x}, \xi) P_{\xi}(\boldsymbol{x} ; t)
$$

in which the evolution operator $\mathcal{L}_{0}(\boldsymbol{x})$ depends only on the variables of interest, while the term $\mathcal{L}_{I}(\boldsymbol{x}, \xi)$ expresses the interaction with the rest of the system. In interaction representation Eq. (11) becomes

$$
\partial_{t} \tilde{P}_{\xi}(\boldsymbol{x} ; t)=\Omega(\boldsymbol{x}, \xi ; t) \tilde{P}_{\xi}(\boldsymbol{x} ; t)
$$

where

$$
\tilde{P}_{\xi}(\boldsymbol{x} ; t):=e^{-\mathcal{L}_{0}(\boldsymbol{x}) t} P_{\xi}(\boldsymbol{x} ; t),
$$

and

$$
\Omega(\boldsymbol{x}, \xi ; t):=e^{-\mathcal{L}_{0}(\boldsymbol{x}) t} \mathcal{L}_{I}(\boldsymbol{x}, \xi) e^{\mathcal{L}_{0}(\boldsymbol{x}) t}
$$

Integrating Eq. (21) and then averaging over the space of the non observable variables $\xi$, we get an equation for the reduced DF:

$$
\tilde{P}(\boldsymbol{x} ; t)=\left\langle\overleftarrow{\exp }\left[\int_{0}^{t} \mathrm{~d} u \Omega(u)\right]\right\rangle P(\boldsymbol{x} ; 0)
$$

in which $\Omega(u):=\Omega(\boldsymbol{x}, \xi ; u)$, $\overleftarrow{\exp }[\ldots]$ is the standard $t$-ordered exponential, $P(\boldsymbol{x} ; t):=$ $\left\langle P_{\xi}(\boldsymbol{x} ; t)\right\rangle$ and we have assumed that $P_{\xi}(\boldsymbol{x} ; t=0)=P(\boldsymbol{x} ; t=0)$ : at the initial time the DF of the variables of interest does not depend on the hidden variables $\xi$. Usually, we aim at obtaining an equation of motion for the reduced DF

$$
\partial_{t} \tilde{P}(\boldsymbol{x} ; t)=\mathfrak{G}(t, \tilde{P}(\boldsymbol{x} ; t))
$$

or, more realistically, a systematic expansion of the local or non local operator $\mathfrak{G}(t, \tilde{P}(\boldsymbol{x} ; t))$, in terms of the fluctuations of the variables over which we have averaged. This can be done formally by writing the solution of Eq. (6) as

$$
\tilde{P}(\boldsymbol{x} ; t)=\sum_{i=0}^{\infty} \mathcal{K}^{(i)}(\boldsymbol{x} ; t) P(\boldsymbol{x} ; 0)
$$

The problem is to find a relation between the operators $\mathcal{K}^{(i)}$ and the series that we obtain by expanding the $t$-ordered exponential of Eq. (5). For that, Kubo $\frac{1}{1}$ started by considering $\Omega(\boldsymbol{x}, \xi ; t)$ as a "random" operator, the statistics of which is determined by the probability 
measure $\mathrm{d} \mu=\rho(\xi) \mathrm{d} \xi$. Given that, the time-evolution operator of Eq. (5) , can be considered as a sort of moment generating function $\mathcal{M}(t)$ for the q-number $\underline{20}$ "stochastic process" $\Omega(t)$ :

$$
\mathcal{M}(t):=\left\langle\overleftarrow{\exp }\left[\int_{0}^{t} \mathrm{~d} u \Omega(u)\right]\right\rangle
$$

The operator $\mathcal{M}(t)$ of Eq. (8) can be written as

$$
\begin{gathered}
\mathcal{M}(t)=\sum_{n=0}^{\infty} \mathcal{M}_{n}(t), \\
\mathcal{M}_{n}(t)=\frac{1}{n !}\left\langle\left\{\left[\int_{0}^{t} \mathrm{~d} u \Omega(u)\right]^{n}\right\}\right\rangle \\
=\frac{1}{n !} \int_{0}^{t} \mathrm{~d} u_{1} \int_{0}^{t} \mathrm{~d} u_{2} \ldots \int_{0}^{t} \mathrm{~d} u_{n}\left\langle\left\{\Omega\left(u_{1}\right) \Omega\left(u_{2}\right) \ldots \Omega\left(u_{n}\right)\right\}_{O}\right\rangle \\
=\int_{0}^{t} \mathrm{~d} u_{1} \int_{0}^{u_{1}} \mathrm{~d} u_{2} \ldots \int_{0}^{u_{n-1}} \mathrm{~d} u_{n}\left\langle\Omega\left(u_{1}\right) \Omega\left(u_{2}\right) \ldots \Omega\left(u_{n}\right)\right\rangle .
\end{gathered}
$$

In Eq. (10) we have introduced the notation $\{\ldots\}_{O}$ that hereafter means "time ordering"; a definition that shall be better specified in the following, but that for the purpose of the present Section can be simply associated to the definition of the $t$-ordered exponential. The average $\left\langle\left\{\Omega\left(u_{1}\right) \Omega\left(u_{2}\right) \ldots \Omega\left(u_{n}\right)\right\}_{O}\right\rangle\left(0 \leq u_{i} \leq t, 1 \leq i \leq n\right)$ is called $t$-ordered $n$-moment density function.

Then, in analogy with c-number (objects of a commutative algebra) stochastic processes, to the moment generating function $\mathcal{M}(t)$, Kubo associates the corresponding cumulant generating function $\mathcal{K}(t)$ (in general, still a q-number):

$$
\exp _{M}[\mathcal{K}]:=\left\langle\overleftarrow{\exp }\left[\int_{0}^{t} \mathrm{~d} u \Omega(u)\right]\right\rangle
$$

Expanding $\mathcal{K}$ in components, $\mathcal{K}(t)=\sum_{n=1}^{\infty} \mathcal{K}_{n}(t)$, and exploiting Eq. (99), we get

$$
\exp _{M}\left[\sum_{n=1}^{\infty} \mathcal{K}_{n}(t)\right]:=\sum_{n=0}^{\infty} \mathcal{M}_{n}(t) .
$$

In the last two equations, $\exp _{M}$ is a generalized exponential, the proper definition and the discussion of which is the central task of the present work. The $\mathcal{K}_{n}$ components are found by expanding the generalized exponential in the 1.h.s. of Eq. (12) and comparing terms of the same order on both sides of the same equation, starting from $n=1$. In this way the analogy with the standard definition for commuting processes seems complete. Remains to 
show how and if this procedure works, and this goes back to the definition of the generalized exponential $\exp _{M}$ introduced in Eqs. (11). As it is well known, the fundamental characteristic of the cumulants is to be vanishing when including independent processes and, for c-number "stochastic variables", this is directly related to the factorization property of the exponential function: $\exp (a+b)=\exp (a) \exp (b)$. To the best of our knowledge, no exponential function extension (actually, no function) shares this property when the arguments are q-numbers, instead of c-numbers. Thus, if $\mathcal{K}$ and $\mathcal{K}^{\prime}$ are non commuting cumulant generating functions, then, in general, the following equation

$$
\exp _{M}[\mathcal{K}] \exp _{M}\left[\mathcal{K}^{\prime}\right]=\exp _{M}\left[\mathcal{K}+\mathcal{K}^{\prime}\right]
$$

does not hold true, whatever the definition of the generalized exponential. This was the main point in Fox ${ }^{6}$ criticism of the general theoretical foundation of Kubo's results. In fact, concerning the factorization property of generalized exponentials of cumulants, he wrote "it is not difficult to show that for $T$ ordering, counter examples $[\ldots]$ can be constructed, and that even though the cluster property is nevertheless true, Kubo's argument does not justify it."

Fox criticized also the Kubo's closed-form formula that gives directly the generalized cumulants in terms of generalized moments. In fact, he wrote: “Eq. (6.9) of Kubo's paper 1 purports to be a closed-form formula for ordered cumulants [...]", but, for him, it is not: "The nature of the error is somewhat subtle, [...] but very significant". Fox stated that in the case where the generalized exponential is the $t$-ordered one, the right procedure to obtain cumulants from moments is that of van Kampen ${ }^{21}, 22$, that he explicitly proved to be different from Kubo's. However, van Kampen ${ }^{21,22}$ and Fox himself, working directly (and heavily) with the analytical expressions of the cumulants defined by the $t$-ordered exponential, demonstrated the "clustering" property of cumulants operators, namely, a special case of the general Kubo's result. Something similar was demonstrated by Terwiel ${ }^{23}$ working with the Zwanzig projection approach. Moreover, van Kampen²1,22 obtained a closed-form formula that gives cumulants in terms of moments, still valid in the special (although important) case of $t$-ordered generalized exponential. In summary, Fox and van Kampen did not consider their results and the one of Terwiel, as particular cases of the Kubo's generalized cumulant theory.

As we have already stressed, the concept of generalized cumulants has been developed 
and applied in many different contexts of physics: dynamical mean-field theory ${ }^{24}$, coupled bosons and valence electrons coupled to plasmons ${ }^{25,26}$, multiple plasmons in photoemis$\operatorname{sion}^{27}$, dynamic perturbations in metals ${ }^{28}$, one-electron Green function $\stackrel{29}{ }$, entanglement between electronic domains and reduced density matrix for coupled electrons in general ${ }^{12} \underline{-19}$, extending Wick theorem in particle physics $\underline{30} \underline{-32}$ and in many field of statistical mechanics $6,7,10,11,21-23,33-39$, just to quote some among many others.

In practice, when the generalized exponential introduced by Kubo is just the time ordered one, the explicit calculus by van Kampen and Fox guarantee the cluster property of the cumulants, namely that cumulants vanish if the variables are "statistically un-connected". In other cases, a specific formalism has been developed to address the generalization of the concept of cumulants to individual problems (e.g., in the already cited work on fermions). On the contrary, Kubo's theory aims to be very general, but the weakness (and, unfortunately, some errors) in the theoretical approach originated some confusion about the proper way to use it (e.g.,,$\frac{10}{}$, where Eqs. (2.14a)-(2.14d) are not correct, or ${ }^{35}$ where Eq.(4.9) is wrong because it is erroneously assumed that if the fluctuating function $\eta(t)$ is Gaussian, then the stochastic operator $\Omega(t)=\eta(t) Q(t)$ is also Gaussian in the Kubo's meaning).

Here we shall re-found the K62-63 approach to cumulants, within a sounded formal scheme. Thus we shall show that, although in general the fundamental Eq. (6.9) of Kubo's paper ${ }^{\underline{1}}$ is at least questionable, the general approach (and idea) is correct. Moreover, we shall give the right relation between moments and cumulants.

In the final section, we will shortly show to apply the present theoretical framework to some noticeable examples.

\section{SOME FORMAL DEFINITIONS AND RESULTS}

Let $\mathfrak{B}$ be a vector space over a field with finite or infinite (typically) dimension. We indicate with $\mathscr{B}=\left\{\mathfrak{b}_{i}\right\},(i \in \mathbb{N}$, or $\mathbb{R})$ a selected basis of $\mathfrak{B}$, that we shall call the "original special basis". For $n \in \mathbb{N}$, let

$$
\mathfrak{B}^{\otimes n}:=\underbrace{\mathfrak{B} \otimes \ldots \otimes \mathfrak{B}}_{n \text { times }}
$$

be the $n$-th tensor power of the $\mathfrak{B}$, of which, for any $n$, the special basis $\mathscr{B}^{\otimes n}$ is the $n$-th tensor powers of $\mathscr{B}$. We indicate with $\mathfrak{M}$ the vector space given by the direct sum of the 
vector spaces $\mathfrak{B}^{\otimes n}$ for $1 \leq n<\infty$ :

$$
\mathfrak{M}:=\mathfrak{B} \oplus \mathfrak{B}^{\otimes 2} \oplus \mathfrak{B}^{\otimes 3} \ldots \oplus \mathfrak{B}^{\otimes k} \oplus \mathfrak{B}^{\otimes(k+1)} \ldots
$$

The special basis $\mathscr{M}$ of $\mathfrak{M}$ is given by the direct sum of the special basis $\mathscr{B}^{\otimes n}$ :

$$
\begin{aligned}
\mathscr{M} & =\left\{\mathfrak{m}_{i}\right\}:=\left\{\mathfrak{m}_{i_{1}, i_{2}, \ldots, i_{n}, \ldots}\right\} \\
& :=\mathscr{B} \oplus \mathscr{B}^{\otimes 2} \oplus \mathscr{B}^{\otimes 3} \ldots \oplus \mathscr{B}^{\otimes k} \oplus \mathscr{B}^{\otimes(k+1)} \ldots
\end{aligned}
$$

with $i_{1}, i_{2}, \ldots, i_{n} \in \mathbb{N}$, or $\mathbb{R}$ and $n \in \mathbb{N}$. We call operators the elements of $\mathfrak{M}$ and, for subsequent use, we have explicitly indicate with $\mathfrak{m}_{\boldsymbol{i}}:=\mathfrak{m}_{i_{1}, i_{2}, \ldots, i_{n}, \ldots}$ the elements of the special basis of $\mathfrak{M}$. For convenience, let's associate also a subscript index (possibly a continuous parameter) to the generic operators: $\mathcal{A}_{i} \in \mathfrak{M}$, such that $\mathcal{A}_{i} \neq \mathcal{A}_{j}$ if $i \neq j$.

Remark A. The tensor product is not, in general, commutative, but, by the universal property, it is associative:

$$
\mathcal{A}_{1} \otimes\left(\mathcal{A}_{2} \otimes \mathcal{A}_{3}\right)=\left(\mathcal{A}_{1} \otimes \mathcal{A}_{2}\right) \otimes \mathcal{A}_{3}
$$

Now we define the mapping that we shall use to introduce the generalized moment generating function.

Definition A. A M-projection $\left\{\mathcal{A}_{i}\right\}_{M}$ is a linear map $\phi_{M}: \mathfrak{M} \rightarrow \mathfrak{M}_{M}$ where $\mathfrak{M}_{M}$ is a subset of $\mathfrak{M}$, such that

(i) the $M$-projection is idempotent, namely, it is a projection map: $\phi_{M} \circ \phi_{M}=\phi_{M}$, or $\left\{\left\{\mathcal{A}_{i}\right\}_{M}\right\}_{M}=\left\{\mathcal{A}_{i}\right\}_{M}$

(ii) the $M$-projection is defined by mapping the elements of the special basis: if $\mathcal{A}_{i}=$ $\sum_{i_{1}, i_{2}, \ldots, i_{n}} a_{i_{1}, i_{2}, \ldots, i_{n}} \mathfrak{m}_{i_{1}, i_{2}, \ldots, i_{n}}$, then $\left\{\mathcal{A}_{i}\right\}_{M}:=\sum_{i_{1}, i_{2}, \ldots, i_{n}} a_{i_{1}, i_{2}, \ldots, i_{n}}\left\{\mathfrak{m}_{i_{1}, i_{2}, \ldots, i_{n}}\right\}_{M}$

(iii) the order of the operators does not matter:

$$
\left\{\mathcal{A}_{1} \otimes \mathcal{A}_{2} \otimes \ldots \otimes \mathcal{A}_{n}\right\}_{M}=\left\{\mathcal{A}_{1^{\prime}} \otimes \mathcal{A}_{2^{\prime}} \otimes \ldots \otimes \mathcal{A}_{n^{\prime}}\right\}_{M}
$$

where $1^{\prime}, 2^{\prime}, \ldots, n^{\prime}$ is any permutation of the indices $1,2, \ldots, n$ (that meas any permutation of the operators inside the brackets); 
Of course, the time ordering defined by the $t$-ordered exponential of Eq. (8) is an $M$ projection map. In physics literature, it is the most common among the $M$-projection maps that correspond to effective ordering respect to some parameters to which depend the operators $\mathcal{A}_{i} \in \mathfrak{M}$. Considering the $M$-projection associated to the $t$-ordered exponential, the point (ii) is strictly related to the following well known surprising "paradox" (see플 where this fact caused confusion and see ${ }^{41}$, instead, for an example where this apparent paradox is properly considered): let $\mathcal{K}(t)$ a linear operator and consider the following trivial identity:

$$
\mathcal{K}(t)-\mathcal{K}(0)=\int_{0}^{t} \mathrm{~d} u \partial_{u} \mathcal{K}(u) .
$$

Now, if we apply the $t$-ordered exponential to both sides of the above equations, the consequent equation

$$
\overleftarrow{\exp }[\mathcal{K}(t)-\mathcal{K}(0)]=\overleftarrow{\exp }\left[\int_{0}^{t} \mathrm{~d} u \partial_{u} \mathcal{K}(u)\right]
$$

does not hold!. The pitfall stays in a wrong association, to the $t$-ordered exponential, of the time ordering operator. The point (ii) of Definition $\mathrm{A}$ says that to apply the $M$-projection ordering map to the l.h.s. of Eq. (17), the operator $\mathcal{K}(t)-\mathcal{K}(0)$ must be expanded in the special basis, that is defined in the r.h.s. of the same equation by the time integral $\int_{0}^{t} \mathrm{~d} u \partial_{u} \mathcal{K}(u)$. See Appendix $\mathrm{A}$ for an explicit example and a clarification about this point.

Besides the time ordering, others examples of $M$-projection maps are, total symmetrization:

$$
\left\{\mathcal{A}_{1} \otimes \mathcal{A}_{2} \otimes \ldots \otimes \mathcal{A}_{n}\right\}_{M}=\frac{1}{n !} \sum_{\text {permutations }} \mathcal{A}_{1} \otimes \mathcal{A}_{2} \otimes \ldots \otimes \mathcal{A}_{n}
$$

or anti-symmetrization, operations that are involved in quantum mechanics of identical particles (see the example in Section IVD 1).

Remark B. By Definition A, point (iii), combining the $M$-projection with the tensor product $\otimes$ we have a commutative operation: $\left\{\mathcal{A}_{1} \otimes \mathcal{A}_{2}\right\}_{M}:=\mathcal{A}_{1} \otimes_{M} \mathcal{A}_{2}=\mathcal{A}_{2} \otimes_{M} \mathcal{A}_{1}$. However, as for time ordering, caution must be taken using this definition of $\otimes_{M}$, because, as it is clear by the points (ii), the $M$-projection severely affects the algebraic structure of the set $\mathfrak{M}$, because the associativity and the distributivity properties does not hold anymore. In particular, in general we have:

$$
\begin{aligned}
& \left\{\mathcal{A}_{1} \otimes \mathcal{A}_{2} \otimes \ldots \otimes \mathcal{A}_{m} \otimes \mathcal{A}_{m+1} \otimes \ldots \otimes \mathcal{A}_{m+n}\right\}_{M} \\
& \neq\left\{\mathcal{A}_{1} \otimes \mathcal{A}_{2} \otimes \ldots \otimes \mathcal{A}_{m}\right\}_{M} \otimes\left\{\mathcal{A}_{m+1} \otimes \ldots \otimes \mathcal{A}_{m+n}\right\}_{M}
\end{aligned}
$$


For example, if the $M$-projection is the total symmetrization operator of Eq. (18), it is clear that while in the 1.h.s. of Eq. (19) we have a totally symmetric result, in the r.h.s. the symmetrization is not complete.

Remark C. Eq. (19) stays at the basis of the Fox criticism 6.7 to the Kubo's theory of cumulants.

Definition B. Given an $M$-projection defined on $\mathfrak{M}$, two operators $\mathcal{A}$ and $\mathcal{B} \in \mathfrak{M}$ are M-unconnected to each other when

$$
\{\mathcal{A} \otimes \mathcal{B}\}_{M}=\{\mathcal{A}\}_{M} \otimes\{\mathcal{B}\}_{M}
$$

Notice, Eq. (20) does not implies $\{\mathcal{A} \otimes \mathcal{B}\}_{M}=\{\mathcal{B}\}_{M} \otimes\{\mathcal{A}\}_{M}$.

For any element $\mathcal{A} \in \mathfrak{M}$ let

$$
\mathcal{A}^{n}:=\underbrace{\mathcal{A} \otimes \ldots \otimes \mathcal{A}}_{n \text { times }}
$$

be the $n$-th tensor power of $\mathcal{A}$. Of course $\mathcal{A}^{n} \in \mathfrak{M}$.

Definition C. If there are two subsets $\mathfrak{M}^{(A)}, \mathfrak{M}^{(B)} \subset \mathfrak{M}$ such that for any operator $\mathcal{A}_{i} \in$ $\mathfrak{M}^{(A)}$ and $\mathcal{B}_{j} \in \mathfrak{M}^{(B)}$, and for any $n_{i}, m_{j}, p, q \in \mathbb{N}$ we have

$$
\begin{aligned}
& \left\{\mathcal{A}_{1}^{n_{1}} \otimes \mathcal{A}_{2}^{n_{2}} \otimes \ldots \otimes \mathcal{A}_{p}^{n_{p}} \otimes \mathcal{B}_{1}^{m_{1}} \otimes \mathcal{B}_{2}^{m_{2}} \otimes \ldots \otimes \mathcal{B}_{q}^{m_{q}}\right\}_{M} \\
= & \left\{\mathcal{A}_{1}^{n_{1}} \otimes \mathcal{A}_{2}^{n_{2}} \otimes \ldots \otimes \mathcal{A}_{p}^{n_{p}}\right\}_{M} \otimes\left\{\mathcal{B}_{1}^{m_{1}} \otimes \mathcal{B}_{2}^{m_{2}} \otimes \ldots \otimes \mathcal{B}_{q}^{m_{q}}\right\}_{M}
\end{aligned}
$$

then the subsets $\mathfrak{M}^{(A)}$ and $\mathfrak{M}^{(B)}$ are called $M$-unconnected to each other.

Remark D. The two subsets $\mathfrak{M}^{(A)}$ and $\mathfrak{M}^{(B)}$ as in Definition $\mathbb{C}$ are vector spaces. In fact

$$
\begin{aligned}
& \left\{\sum_{i} \mathcal{A}_{i} \otimes \sum_{j} \mathcal{B}_{j}\right\}_{M}=\sum_{i, j}\left\{\mathcal{A}_{i} \otimes \mathcal{B}_{j}\right\}_{M} \\
& =\sum_{i, j}\left\{\mathcal{A}_{i}\right\}_{M} \otimes\left\{\mathcal{B}_{j}\right\}_{M}=\left\{\sum_{i} \mathcal{A}_{i}\right\}_{M} \otimes\left\{\sum_{j} \mathcal{B}_{j}\right\}_{M}
\end{aligned}
$$

thus we shall use the therm M-unconnected "subspaces" instead of M-unconnected subsets. Moreover, by Eq. (21) we see that the two unconnected sets can be infinitely extended by including all the possible powers of their original elements.

Remark E. In the r.h.s. of Eq. (21), the ordering between the $\mathcal{A}_{i}$ and the $\mathcal{B}_{j}$ elements is usually important (here, the $\mathcal{A}_{i}$ stay at the left). 
Proposition 1. In Definition (C), we can substitute Eq. (21) with the following equivalent one:

$$
\left\{\mathcal{A}_{i}^{n} \otimes \mathcal{B}_{j}^{m}\right\}_{M}=\left\{\mathcal{A}_{i}^{n}\right\}_{M} \otimes\left\{\mathcal{B}_{j}^{m}\right\}_{M} \quad \forall n, m \in \mathbb{N} .
$$

At first sight the condition given in Eq. (22) looks less restrictive than that of Eq. (21), however, the equivalence can be demonstrated by using the fact that the sets $\mathfrak{M}^{(A)}$ and $\mathfrak{M}^{(B)}$ are infinite dimensional vector spaces:

Proof of Proposition 1. To demonstrate the proposition, let us write the operators $\mathcal{A}_{i}$ and $\mathcal{B}_{j}$ in terms of the elements of the special basis of $\mathfrak{M}^{(A)}$ and $\mathfrak{M}^{(B)}$, respectively:

$$
\begin{aligned}
\mathcal{A}_{i} & =\sum_{k=1}^{p} a_{i_{k}} \mathfrak{m}_{i_{k}} \\
\mathcal{B}_{j} & =\sum_{h=1}^{q} b_{j_{h}} \mathfrak{m}_{j_{h}}
\end{aligned}
$$

inserting these expansions in Eq. (22) we get:

$$
\begin{aligned}
& \left\{\left(\sum_{k=1}^{p} a_{\boldsymbol{i}_{k}} \mathfrak{m}_{\boldsymbol{i}_{k}}\right)^{n} \otimes\left(\sum_{h=1}^{q} b_{\boldsymbol{j}_{h}} \mathfrak{m}_{j_{h}}\right)^{m}\right\}_{M} \\
& =\left\{\left(\sum_{k=1}^{p} a_{\boldsymbol{i}_{k}} \mathfrak{m}_{\boldsymbol{i}_{k}}\right)^{n}\right\}_{M} \otimes\left\{\left(\sum_{h=1}^{q} b_{\boldsymbol{j}_{h}} \mathfrak{m}_{\boldsymbol{j}_{h}}\right)^{m}\right\}_{M},
\end{aligned}
$$

by using the multinomial theorem the above equation becomes $\left(r_{k}\right.$ and $s_{h}$ are such that $\sum_{k=1}^{p} r_{k}=n$ and $\sum_{h=1}^{q} s_{h}=m$, respectively)

$$
\begin{aligned}
& \sum_{r_{k}} \sum_{s_{h}} \frac{n !}{r_{1} ! r_{2} ! \ldots r_{p}} \frac{m !}{s_{1} ! s_{2} ! \ldots s_{q}} a_{\boldsymbol{i}_{1}}^{r_{1}} a_{\boldsymbol{i}_{2}}^{r_{2}} \ldots a_{\boldsymbol{i}_{r_{p}}}^{r_{p}} b_{\boldsymbol{j}_{1}}^{s_{1}} b_{\boldsymbol{j}_{2}}^{s_{2}} \ldots b_{\boldsymbol{j}_{s_{q}}}^{s_{q}} \\
& \times\left\{\mathfrak{m}_{\boldsymbol{i}_{1}}^{r_{1}} \otimes \mathfrak{m}_{i_{2}}^{r_{2}} \otimes \ldots \mathfrak{m}_{\boldsymbol{i}_{p}}^{r_{p}} \otimes \mathfrak{m}_{j_{1}}^{s_{1}} \otimes \mathfrak{m}_{j_{2}}^{s_{2}} \otimes \ldots \mathfrak{m}_{j_{q}}^{r_{q}}\right\}_{M} \\
& =\sum_{r_{k}} \sum_{s_{h}} \frac{n !}{r_{1} ! r_{2} ! \ldots r_{p}} \frac{m !}{s_{1} ! s_{2} ! \ldots s_{q}} a_{\boldsymbol{i}_{1}}^{r_{1}} a_{\boldsymbol{i}_{2}}^{r_{2}} \ldots a_{\boldsymbol{i}_{r_{p}}}^{r_{p}} b_{\boldsymbol{j}_{1}}^{s_{1}} b_{\boldsymbol{j}_{2}}^{s_{2}} \ldots b_{\boldsymbol{j}_{s_{q}}}^{s_{q}} \\
& \times\left\{\mathfrak{m}_{\boldsymbol{i}_{1}}^{r_{1}} \otimes \mathfrak{m}_{\boldsymbol{i}_{2}}^{r_{2}} \otimes \ldots \mathfrak{m}_{\boldsymbol{i}_{p}}^{r_{p}}\right\}_{M} \otimes\left\{\mathfrak{m}_{\boldsymbol{j}_{1}}^{s_{1}} \otimes \mathfrak{m}_{j_{2}}^{s_{2}} \otimes \ldots \mathfrak{m}_{j_{q}}^{r_{q}}\right\}_{M}
\end{aligned}
$$

from which,

$$
\begin{aligned}
& \sum_{r_{k}} \sum_{s_{h}} \frac{n !}{r_{1} ! r_{2} ! \ldots r_{p}} \frac{m !}{s_{1} ! s_{2} ! \ldots s_{q}} a_{i_{1}}^{r_{1}} a_{\boldsymbol{i}_{2}}^{r_{2}} \ldots a_{\boldsymbol{i}_{r_{p}}}^{r_{p}} b_{j_{1}}^{s_{1}} b_{\boldsymbol{j}_{2}}^{s_{2}} \ldots b_{j_{s_{q}}}^{s_{q}} \\
& \times\left(\left\{\mathfrak{m}_{i_{1}}^{r_{1}} \otimes \mathfrak{m}_{i_{2}}^{r_{2}} \otimes \ldots \mathfrak{m}_{i_{p}}^{r_{p}} \otimes \mathfrak{m}_{j_{1}}^{s_{1}} \otimes \mathfrak{m}_{j_{2}}^{s_{2}} \otimes \ldots \mathfrak{m}_{j_{q}}^{r_{q}}\right\}_{M}\right. \\
& \left.-\left\{\mathfrak{m}_{i_{1}}^{r_{1}} \otimes \mathfrak{m}_{i_{2}}^{r_{2}} \otimes \ldots \mathfrak{m}_{i_{p}}^{r_{p}}\right\}_{M} \otimes\left\{\mathfrak{m}_{j_{1}}^{s_{1}} \otimes \mathfrak{m}_{j_{2}}^{s_{2}} \otimes \ldots \mathfrak{m}_{j_{q}}^{r_{q}}\right\}_{M}\right) \\
& =0 .
\end{aligned}
$$


Because the vector spaces $\mathfrak{M}^{(A)}, \mathfrak{M}^{(B)}$ are infinite-dimensional, $n$ and $m$ can be any integer, thus the same it holds for all the powers $r_{k}, s_{h}$. Considering that the coefficients $a_{\boldsymbol{i}_{k}}$ and $b_{\boldsymbol{j}_{h}}$ are arbitrary, it follows that Eq. (26) implies

$$
\begin{aligned}
& \left\{\mathfrak{m}_{\boldsymbol{i}_{1}}^{r_{1}} \otimes \mathfrak{m}_{\boldsymbol{i}_{2}}^{r_{2}} \otimes \ldots \mathfrak{m}_{\boldsymbol{i}_{p}}^{r_{p}} \otimes \mathfrak{m}_{\boldsymbol{j}_{1}}^{s_{1}} \otimes \mathfrak{m}_{\boldsymbol{j}_{2}}^{s_{2}} \otimes \ldots \mathfrak{m}_{\boldsymbol{j}_{q}}^{r_{q}}\right\}_{M} \\
& =\left\{\mathfrak{m}_{\boldsymbol{i}_{1}}^{r_{1}} \otimes \mathfrak{m}_{\boldsymbol{i}_{2}}^{r_{2}} \otimes \ldots \mathfrak{m}_{\boldsymbol{i}_{p}}^{r_{p}}\right\}_{M} \otimes\left\{\mathfrak{m}_{\boldsymbol{j}_{1}}^{s_{1}} \otimes \mathfrak{m}_{\boldsymbol{j}_{2}}^{s_{2}} \otimes \ldots \mathfrak{m}_{\boldsymbol{j}_{q}}^{r_{q}}\right\}_{M}
\end{aligned}
$$

$\forall r_{k}, s_{h} \in \mathbb{N}, p, q \in \mathbb{N}$, which, in turn, implies Eq (21).

If $f(x)$ is an analytical function in the argument $x$ and $f^{(n)}(x):=\mathrm{d}^{n} / \mathrm{d} x^{n} f(x)$, then we can define $\left.f(\mathcal{A}):=\sum_{n=0}^{\infty} \frac{1}{n !} f^{(n)}(0)\right) \mathcal{A}^{n}$. Thus, we can easily define the generalized exponential for non commuting operators:

Definition D. For any $\mathcal{A} \in \mathfrak{M}$ the $M$-exponential $\exp _{M}[\mathcal{A}]$ is defined as the $M$-projection applied to the formal tensor power expansion of $\exp [\mathcal{A}]$ :

$$
\begin{aligned}
\exp _{M}[\mathcal{A}] & :=\{\exp [\mathcal{A}]\}_{M}=\left\{\sum_{n=0}^{\infty} \frac{1}{n !} \mathcal{A}^{n}\right\}_{M} \\
& =\sum_{n=0}^{\infty} \frac{1}{n !}\left\{\mathcal{A}^{n}\right\}_{M}
\end{aligned}
$$

Theorem 1. Let $\mathcal{A}_{i} \in \mathfrak{M}, 1 \leq i \leq N$, then the following factorization property holds:

$$
\exp _{M}\left[\sum_{i=1}^{N} \mathcal{A}_{i}\right]=\left\{\prod_{i=1}^{N} \exp \left[\mathcal{A}_{i}\right]\right\}_{M} .
$$

Proof of Theorem 1. The demonstration is trivial because by the definition of the $M$ projection, properties (iii), the argument of the $M$-projection can be formally freely rearranged, without caring about the order of the factors, thus the multinomial theorem can be used

$$
\begin{aligned}
& \exp _{M}\left[\sum_{i=1}^{N} \mathcal{A}_{i}\right]:=\sum_{n=0}^{\infty} \frac{1}{n !}\left\{\left[\sum_{i=1}^{N} \mathcal{A}_{i}\right]^{n}\right\}_{M} \\
& =\sum_{m_{1}=0}^{\infty} \sum_{m_{2}=0}^{\infty} \ldots \sum_{m_{N}=0}^{\infty} \frac{1}{m_{1} !} \frac{1}{m_{2} !} \cdot \frac{1}{m_{N} !} \\
& \times\left\{\mathcal{A}_{1}^{m_{1}} \otimes \mathcal{A}_{2}^{m_{2}} \otimes \ldots \otimes \mathcal{A}_{N}^{m_{N}}\right\}_{M}=\left\{\prod_{i=1}^{N} \exp \left[\mathcal{A}_{i}\right]\right\}_{M} .
\end{aligned}
$$

Remark F. Although Theorem 1 shows that the $M$-exponential shares a fundamental property with the usual exponential, this fact does not imply that Eq. (13) holds true. We think 
that this is the principal source of confusion in the work of Kubo ${ }^{1}$, and the basis of the Fox criticism. However, for a proper definition of cumulants, Eq. (13) is not required to hold in general, but only when the processes $\Omega(u), u \in T$ and $\Omega\left(u^{\prime}\right), u^{\prime} \in T^{\prime}$, to which the generating cumulant functions $\mathcal{K}$ and $\mathcal{K}^{\prime}$, respectively, are related, are "independent" to each other. As we shall show in the next section, with a proper definition of independent stochastic processes, this is the case, thanks to the following result:

Theorem 2. Two vector subspaces $\mathfrak{M}^{(A)}, \mathfrak{M}^{(B)} \subset \mathfrak{M}$, are $M$-unconnected to each other, if and only if, for any $\mathcal{A} \in \mathfrak{M}^{(A)}$ and $\mathcal{B} \in \mathfrak{M}^{(B)}$ we have:

$$
\begin{aligned}
& \exp _{M}[\mathcal{A}+\mathcal{B}]=\exp _{M}[\mathcal{A}] \otimes \exp _{M}[\mathcal{B}] \text { or } \\
& \exp _{M}[\mathcal{A}+\mathcal{B}]=\exp _{M}[\mathcal{B}] \otimes \exp _{M}[\mathcal{A}]
\end{aligned}
$$

Proof of Theorem 2. According to Definition D, the r.h.s of Eq. (31) can be rewritten as (let's consider only the ordering given in the first line, for simplicity):

$$
\begin{aligned}
& \exp _{M}[\mathcal{A}] \otimes \exp _{M}[\mathcal{B}]:=\sum_{n=0}^{\infty} \frac{1}{n !}\left\{\mathcal{A}^{n}\right\}_{M} \otimes \sum_{m=0}^{\infty} \frac{1}{m !}\left\{\mathcal{B}^{m}\right\}_{M} \\
& =\sum_{n, m=0}^{\infty} \frac{1}{n !} \frac{1}{m !}\left\{\mathcal{A}^{n}\right\}_{M} \otimes\left\{\mathcal{B}^{m}\right\}_{M},
\end{aligned}
$$

while from Theorem 1 the l.h.s. is given by

$$
\exp _{M}[\mathcal{A}+\mathcal{B}]=\sum_{n, m=0}^{\infty} \frac{1}{n !} \frac{1}{m !}\left\{\mathcal{A}^{n} \otimes \mathcal{B}^{m}\right\}_{M}
$$

Comparing Eq. (32) with Eq. (33), exploiting Definition Cof $M$-unconnected sets and considering that $\mathcal{A}$ and $\mathcal{B}$ are generic elements of $\mathfrak{M}^{(A)}$ and $\mathfrak{M}^{(B)}$, respectively, it follows that if $\mathfrak{M}^{(A)}$ and $\mathfrak{M}^{(B)}$ are $M$-unconnected, then Eq. (31) holds. The reverse follows straightforward from Proposition 1.

Now we introduce the map that we shall use to define the generalized cumulants:

Definition E. an $M$-ordering map is an $M$-projection map subjected to the condition that if for some $\mathcal{A}, \mathcal{B} \in \mathfrak{M}$ we have $\{\mathcal{A}\}_{M} \otimes\{\mathcal{B}\}_{M}=\left\{\{\mathcal{A}\}_{M} \otimes\{\mathcal{B}\}_{M}\right\}_{M}$ (thus, $\{\mathcal{A}\}_{M} \otimes\{\mathcal{B}\}_{M} \in$ $\mathfrak{M}_{M}$, the co-domain of the $M$-projection map) then $\{\mathcal{A}\}_{M} \otimes\{\mathcal{B}\}_{M}=\{\mathcal{A} \otimes \mathcal{B}\}_{M}$ (namely, $\mathcal{A}$ and $\mathcal{B}$ are $M$-unconnected to each other). 
It is clear that an $M$-projection map corresponding to some ordering/symmetrization respect to some index/parameter labeling the operators of $\mathfrak{M}$ is an $M$-ordering map. However, in general an $M$-projection map involving some average procedure is not an $M$-ordering map. For example, if some subspace $\mathfrak{M}^{(A)}$ of $\mathfrak{M}$ is spanned by the parameters $i \in I_{i}, y \in I_{y}$, $I_{i} \subset \mathbb{N}, I_{y} \subset \mathbb{R}$ (or $\mathbb{N}$ ), namely, if $\forall i \in I_{i}, y \in I_{y}$ we have $\mathcal{A}(i, y) \in \mathfrak{M}^{(A)}$, then, the $M$ projection map defined as the combined action of some ordering respect to the $i$ parameter (e.g. $i$ decreasing from left to right) and a sum (or average) respect to the $y$ one:

$$
\begin{aligned}
& \left\{\mathcal{A}\left(i_{1}, y_{1}\right) \otimes \mathcal{A}\left(i_{2}, y_{2}\right) \otimes \ldots \otimes \mathcal{A}\left(i_{n}, y_{n}\right)\right\}_{M} \\
& =\int_{I_{y}}\left\{\mathcal{A}\left(i_{1}, y\right) \otimes \mathcal{A}\left(i_{2}, y\right) \otimes \ldots \otimes \mathcal{A}\left(i_{n}, y\right)\right\}_{O_{i}} \mathrm{~d} y
\end{aligned}
$$

is not an $M$-ordering map. In fact, $\{\mathcal{A}\}_{M} \otimes\{\mathcal{B}\}_{M}=\left\{\{\mathcal{A}\}_{M} \otimes\{\mathcal{B}\}_{M}\right\}_{M}$ is satisfied for any $\mathcal{A}=\mathcal{A}\left(i_{1}, y_{1}\right) \otimes \mathcal{A}\left(i_{2}, y_{2}\right) \ldots$ and $\mathcal{B}=\mathcal{A}\left(j_{1}, y_{1}\right) \otimes \mathcal{A}\left(j_{2}, y_{2}\right) \ldots$ such that $i_{k}>j_{h} \forall h, k$, but $\{\mathcal{A}\}_{M} \otimes\{\mathcal{B}\}_{M} \neq\{\mathcal{A} \otimes \mathcal{B}\}_{M}$ (in general, the product of averages is not equal to the average of the product).

In the following the $M$-ordering map applied to a generic operator $\mathcal{A} \in \mathfrak{M}$ shall be indicated by $\{\mathcal{A}\}_{M_{O}}$.

The following result follows now without need of demonstration:

Lemma 1. Let $\mathfrak{M}^{(A)}$ and $\mathfrak{M}^{(B)} \subset \mathfrak{M}$. If $\forall \mathcal{A}_{i} \in \mathfrak{M}^{(A)}$ and $\forall \mathcal{B}_{j} \in \mathfrak{M}^{(B)}$ we have $\left(n_{i}, m_{i}, p, q \in\right.$ $\mathbb{N})$ :

$$
\begin{aligned}
& \left\{\mathcal{A}_{1}^{n_{1}} \otimes \mathcal{A}_{2}^{n_{2}} \otimes \ldots \otimes \mathcal{A}_{p}^{n_{p}}\right\}_{M_{O}} \otimes\left\{\mathcal{B}_{1}^{n_{1}} \otimes \mathcal{B}_{2}^{m_{2}} \otimes \ldots \otimes \mathcal{B}_{q}^{m_{q}}\right\}_{M_{O}} \\
= & \left\{\left\{\mathcal{A}_{1}^{n_{1}} \otimes \mathcal{A}_{2}^{n_{2}} \otimes \ldots \otimes \mathcal{A}_{p}^{n_{p}}\right\}_{M_{O}} \otimes\left\{\mathcal{B}_{1}^{n_{1}} \otimes \mathcal{B}_{2}^{m_{2}} \otimes \ldots \otimes \mathcal{B}_{q}^{m_{q}}\right\}_{M_{O}}\right\}_{M_{O}},
\end{aligned}
$$

then $\mathfrak{M}^{(A)}$ and $\mathfrak{M}^{(B)}$ are $M$-unconnected to each other vector spaces.

\section{THE CASE OF CUMULANTS}

Having defined in a proper way the $M$-exponential, we proceed now with a less formal pathway.

\section{A. General settings}

In the definition of the moment generating function of Eqs. (8)-(10) we have used the usual $t$-ordered exponential. As already observed, the $M$-projection map associated with the 
$t$-ordered exponential, fulfills both the requirements (i) (iii) of the definition of $M$-projection and also the more restrictive Definition E of $M$-ordering. To identify this specific mapping we use the notation $M=O$, namely $\exp _{O}[\ldots]:=\overleftarrow{\exp }[\ldots]$. We generalize Eqs. (8) and (10) by substituting the $t$-ordered exponential with another $M$-exponential associated to a general $M$-projection map, that we shortly indicate with the $M$ map.

Definition F. Assuming that $\Omega$ is a random q-number means here that in the set of the possible "instances" $\Omega$ we have defined a projection map, that we call "average operation (or process)" and that we indicate with $\langle. .$.$\rangle . Of course, the above definition does not coincide$ with the usual one for random numbers, in fact $\Omega$ could be a deterministic (instead of really random) operator that depends on some parameter/variables $\xi$ and $\langle\ldots\rangle$ could correspond to the integration of $\xi$ by using some defined measure $d \mu(\xi)$ (see the example of Eqs. (11)-(4)).

The generalized cumulants that concern this work are related to the definition of independent processes through the factorization of the generalized moment generating function. Namely,

Definition G. if $\Omega_{1}$ and $\Omega_{2}$ are two independent random q-numbers with generalized moment generating functions $\mathcal{M}_{1}:=\left\langle\exp _{M}\left[\Omega_{1}\right]\right\rangle$ and $\mathcal{M}_{2}:=\left\langle\exp _{M}\left[\Omega_{2}\right]\right\rangle$, respectively, then $\Omega_{1}$ and $\Omega_{2}$ are defined statistically independent if and only if the generalized moment generating function $\mathcal{M}_{1 \oplus 2}:=\left\langle\exp _{M}\left[\Omega_{1} \oplus \Omega_{2}\right]\right\rangle$ of the random q-number $\Omega_{1} \oplus \Omega_{2}$ is given by $\mathcal{M}_{1} \otimes \mathcal{M}_{2}$.

Now we introduce some notations and comments for further use:

1. $\mathcal{U}$ is some (generally continuous) set of values for the parameter $u$ and, for any fixed value of $u, \Omega(u)$ is a random operator, namely $\Omega(u)$ is a q-number (or operator) stochastic process. The parameter $u$ does not need to be a time, it could be a temperature value, as in classical and quantum statistical mechanics, or some other index or parameter for the set of stochastic operators $\Omega(u)$.

2. By point 1, a value of $u \in \mathcal{U}$ does not uniquely individuate an operator $\Omega(u$ ) (for example, in the case of Eq. (1)-(4), the $\Omega(u)=\Omega(\boldsymbol{x}, \xi ; u)$ operator depends on the time $u$, but also on the set of variables $\xi$ that we integrate in the average process). We assume that the set of all the possible instances $\Omega$ are the original special basis $\mathscr{B}=$ $\left\{\mathfrak{b}_{i}\right\}$ of a vector space $\mathfrak{B}$, and the tensor product $\otimes$ by which a space $\mathfrak{M}$ can be defined 
(see the beginning of section III) is the product among the operators $\Omega$. From now on, we shall denote with " $M$ map"an $M$-projection defined on this $\mathfrak{M}$ space. Exploiting again the example of Eq. (11)-(44), for any $u \in \mathcal{U}$, and $\xi$ ranging in a proper space where it is defined, we have $\Omega(\boldsymbol{x}, \xi ; u) \in \mathscr{B}$, and $\Omega\left(\boldsymbol{x}, \xi^{\prime} ; u_{1}\right) \Omega\left(\boldsymbol{x}, \xi^{\prime \prime} ; u_{2}\right) \Omega\left(\boldsymbol{x}, \xi^{\prime \prime \prime} ; u_{3}\right) \ldots \in \mathscr{M}$.

3. Because the average process $\langle\ldots\rangle$ is a projection, the composition of an $M$-projection map $M$ with the average operation is, in turn, an $M$-projection map, that we indicate with $\tilde{M}:\{\ldots\}_{\tilde{M}}:=\langle\ldots\rangle \circ\{\ldots\}_{M}($ or, shortly, $\tilde{M}=\langle.\rangle \circ M)$.

4. The generalized moment generating function $\mathcal{M}(\mathcal{T})$ is defined as

$$
\mathcal{M}(\mathcal{T}):=\left\langle\exp _{M}\left[\int_{\mathcal{T}} \mathrm{d} u \Omega(u)\right]\right\rangle:=\exp _{\tilde{M}}\left[\int_{\mathcal{T}} \mathrm{d} u \Omega(u)\right] .
$$

where $\mathcal{T}$ is a subset of $\mathcal{U}$.

5. The generalized moment generating components $\mathcal{M}_{n}(\mathcal{T})$ are given by

$$
\begin{aligned}
& \mathcal{M}_{n}(\mathcal{T})=\frac{1}{n !}\left\{\left[\int_{\mathcal{T}} \mathrm{d} u \Omega(u)\right]^{n}\right\}_{\tilde{M}} \\
& =\frac{1}{n !} \int_{\mathcal{T}} \mathrm{d} u_{1} \int_{\mathcal{T}} \mathrm{d} u_{2} \ldots \int_{\mathcal{T}} \mathrm{d} u_{n}\left\{\Omega\left(u_{1}\right) \Omega\left(u_{2}\right) \ldots \Omega\left(u_{n}\right)\right\}_{\tilde{M}} .
\end{aligned}
$$

Of course we have $\mathcal{M}(\mathcal{T}):=\sum_{n=0}^{\infty} \mathcal{M}_{n}(\mathcal{T})$ as in Eq. (9). From the operative side, the last side of Eq. (36) is mandatory because of the point (ii) of the definition of $M$-projection map.

6. The generalized $n$-moment density function is defined as

$$
\mu_{n}^{(\tilde{M})}\left(u_{1}, u_{2}, \ldots, u_{n}\right):=\left\{\Omega\left(u_{1}\right) \Omega\left(u_{2}\right) \ldots \Omega\left(u_{n}\right)\right\}_{\tilde{M}},
$$

thus, the generalized $n$-moments density functions are elements of the co-domain of the $\tilde{M}$ map, and, in particular, each one corresponds to the mapping of a different element of the special basis $\mathscr{M}$ of the space $\mathfrak{M}$ where the $\tilde{M}$ map is defined.

According to Definition G, we specify what we mean for independence of stochastic operator processes:

Definition H. If $\mathcal{T}$ and $\mathcal{T}^{\prime}$ are two different subsets of $\mathcal{U}$, given the Definition $G$ and the Theorem 2, we say that two q-number stochastic processes $\Omega(u)$ and $\Omega\left(u^{\prime}\right) \in \mathfrak{B}$, with $u \in \mathcal{T}$, $u^{\prime} \in \mathcal{T}^{\prime}$, are $\mathfrak{u}$-independent to each other if the subsets $\mathcal{T}$ and $\mathcal{T}^{\prime}$ define two vector spaces that are $M$-unconnected to each other respect to the $\tilde{M}$ map. 
Remark G. According to Theorem 圆, from Definition $\mathbb{H}$ it follows that the subsets $\mathcal{T}$ and $\mathcal{T}^{\prime}$ are $\mathfrak{u}$-independent to each other if and only if the following factorization of the moment generating function holds true:

$$
\exp _{\tilde{M}}\left[\int_{\mathcal{T} \cup \mathcal{T}^{\prime}} d u \Omega(u)\right]=\exp _{\tilde{M}}\left[\int_{\mathcal{T}} d u \Omega(u)\right] \exp _{\tilde{M}}\left[\int_{\mathcal{T}^{\prime}} d u \Omega(u)\right] .
$$

Having generalized the definition of the moment generating function by introducing the $\tilde{M}$ mapping, we have now to generalize also the definition of the cumulant generating function. For reasons that will become clear later, this is done by using an $M$-ordering map, instead of a more general $M$-projection map $\underline{42}$ :

$$
\exp _{M_{O}}[\mathcal{K}(\mathcal{T})]:=\mathcal{M}(\mathcal{T}):=\exp _{\tilde{M}}\left[\int_{\mathcal{T}} \mathrm{d} u \Omega(u)\right],
$$

in which $M_{O}$ is the $M$-ordering map (the " $M_{O}$ " map, shortly) that applies to the generalized cumulants. Notice that often both the the $M_{O}$ and the $M$ maps are the chronological time ordering that give rise to the $t$-ordered exponential, but in general they can be different to each other (see below, around Eqs. (44)-(45)). According to Kubo $\underline{1}$ the generalized cumulant generating function $\mathcal{K}(\mathcal{T})$ can be conveniently written as

$$
\mathcal{K}(\mathcal{T})=\left\langle\exp _{M^{\prime}}\left[\int_{\mathcal{T}} \mathrm{d} u \Omega(u)\right]-1\right\rangle_{c}
$$

in which $M^{\prime}$ is some other $M$-projection map we shall identify hereafter. Eq. (40) at the same time defines the "cumulant average" $\langle\ldots\rangle_{c}$, and gives rise to the series $\mathcal{K}=\sum_{n=1}^{\infty} \mathcal{K}_{n}$ of cumulant generating components, of which the $n$-th term is

$$
\begin{aligned}
\mathcal{K}_{n} & :=\frac{1}{n !} \int_{\mathcal{T}} \mathrm{d} u_{1} \int_{\mathcal{T}} \mathrm{d} u_{2} \ldots \int_{\mathcal{T}} \mathrm{d} u_{n}\left\langle\left\{\Omega\left(u_{1}\right) \Omega\left(u_{2}\right) \ldots \Omega\left(u_{n}\right)\right\}_{M^{\prime}}\right\rangle_{c} \\
& :=\frac{1}{n !} \int_{\mathcal{T}} \mathrm{d} u_{1} \int_{\mathcal{T}} \mathrm{d} u_{2} \ldots \int_{\mathcal{T}} \mathrm{d} u_{n}\left\{\Omega\left(u_{1}\right) \Omega\left(u_{2}\right) \ldots \Omega\left(u_{n}\right)\right\}_{\tilde{M}_{c}^{\prime}} .
\end{aligned}
$$

where, extending a little the already introduced convention, $\tilde{M}_{c}^{\prime}=\langle.\rangle_{c} \circ M^{\prime}$. In the Kubo's papers K62-63 there is not an indication about the relations among the $M, M_{O}$ and $M^{\prime}$ maps. We shall remedy hereafter to that. It is apparent that if in the l.h.s. of Eq. (39) we take the first order on the power series that defines the $M_{O}$ exponential and if we exploit Eq. (40), for consistency we must have $M^{\prime}=M$. Thus

$$
\begin{aligned}
\mathcal{K}_{n} & :=\frac{1}{n !} \int_{\mathcal{T}} \mathrm{d} u_{1} \int_{\mathcal{T}} \mathrm{d} u_{2} \ldots \int_{\mathcal{T}} \mathrm{d} u_{n}\left\langle\left\{\Omega\left(u_{1}\right) \Omega\left(u_{2}\right) \ldots \Omega\left(u_{n}\right)\right\}_{M}\right\rangle_{c} \\
& :=\frac{1}{n !} \int_{\mathcal{T}} \mathrm{d} u_{1} \int_{\mathcal{T}} \mathrm{d} u_{2} \ldots \int_{\mathcal{T}} \mathrm{d} u_{n}\left\{\Omega\left(u_{1}\right) \Omega\left(u_{2}\right) \ldots \Omega\left(u_{n}\right)\right\}_{\tilde{M}_{c}}
\end{aligned}
$$


The generalized $n$-cumulant density function is then defined as

$$
\kappa_{n}^{\left(\tilde{M}, M_{O}\right)}\left(u_{1}, u_{2}, \ldots, u_{n}\right):=\left\{\Omega\left(u_{1}\right) \Omega\left(u_{2}\right) \ldots \Omega\left(u_{n}\right)\right\}_{\tilde{M}_{c}} .
$$

Remark H. The generalized cumulant density functions of Eq. (43) are the "original special basis" $\mathscr{B}=\left\{\mathfrak{b}_{i}\right\}$ (possible redundant) of the vector space $\mathfrak{B}$, that, togheter with the tensor product $\otimes$, gives rise to the space $\mathfrak{M}$ where the $M_{O}$ map is defined.

By Eq. (43), the generalized $n$-cumulant density functions are elements of the co-domain of the $\tilde{M}_{c}$ map.

Because $\{\ldots\}_{M_{O}}$ is a projection (namely, $\left\{\{\ldots\}_{M_{O}}\right\}_{M_{O}}=\{\ldots\}_{M_{O}}$ ), we can apply the $M_{O}$ map to the l.h.s. of Eq. (39) without affecting the result, thus the same should hold for the r.h.s. of the same equation. Then, using Definition $\mathrm{D}$ we have

$$
\left\{\left(\int_{\mathcal{T}} \mathrm{d} u \Omega(u)\right)^{n}\right\}_{\tilde{M}}=\left\{\left\{\left(\int_{\mathcal{T}} \mathrm{d} u \Omega(u)\right)^{n}\right\}_{\tilde{M}}\right\}_{M_{O}} .
$$

The above equation is the constraint we have to take into account when in choosing the $M_{O}$ map once the $\tilde{M}$ one is given (as is common in real problems), namely, it is the relationship between these two mappings we were looking for. In practice, exploiting Eqs. (36)-(37), Eq. (44) says that the generalized moments must not be affected by the $M_{O}$ map (they belong to the co-domain of $M_{O}$ ):

$$
\begin{aligned}
\left\{\mu_{n}^{(\tilde{M})}\left(u_{1}, u_{2}, \ldots, u_{n}\right)\right\}_{M_{O}} & =\left\{\left\{\Omega\left(u_{1}\right) \Omega\left(u_{2}\right) \ldots \Omega\left(u_{n}\right)\right\}_{\tilde{M}}\right\}_{M_{O}} \\
& =\left\{\Omega\left(u_{1}\right) \Omega\left(u_{2}\right) \ldots \Omega\left(u_{n}\right)\right\}_{\tilde{M}}=\mu_{n}^{(\tilde{M})}\left(u_{1}, u_{2}, \ldots, u_{n}\right) .
\end{aligned}
$$

Of course, the most trivial way to satisfy Eq. (45) is to choose $M_{O} \equiv M$ (the equivalence symbol is used, instead of the equality one, because the $\mathfrak{M}$ spaces of the two mappings are not the same), but we stress again that this is not the only possible choice (e.g., see Section IV D 3).

By using both Eq. (401) and the definition of the $\tilde{M}_{c}$ map, Eq. (39) can be also written as:

$$
\exp _{M_{O}}\left[\exp _{\tilde{M}_{c}}\left[\int_{\mathcal{T}} \mathrm{d} u \Omega(u)\right]-1\right]=\exp _{\tilde{M}}\left[\int_{\mathcal{T}} \mathrm{d} u \Omega(u)\right]
$$

If $M=O$, namely if it is the usual time ordering map (increasing time from right to left), we have

$$
\left.\begin{array}{l}
\mu_{n}^{(\tilde{O})}\left(u_{1}, u_{2}, \ldots, u_{n}\right)=\left\langle\Omega\left(u_{1}\right) \Omega\left(u_{2}\right) \ldots \Omega\left(u_{n}\right)\right\rangle \\
\kappa_{n}^{\left(\tilde{O}, M_{O}\right)}\left(u_{1}, u_{2}, \ldots, u_{n}\right)=\left\langle\Omega\left(u_{1}\right) \Omega\left(u_{2}\right) \ldots \Omega\left(u_{n}\right)\right\rangle_{c}
\end{array}\right\} \text { with } u_{1} \geq u_{2} \geq \ldots \geq u_{n}
$$


that, inserted in Eqs. (36) and (42) gives,

$$
\begin{aligned}
\mathcal{M}_{n} & :=\int_{0}^{t} \mathrm{~d} u_{1} \int_{0}^{u_{1}} \mathrm{~d} u_{2} \ldots \int_{0}^{u_{n-1}} \mathrm{~d} u_{n}\left\langle\Omega\left(u_{1}\right) \Omega\left(u_{2}\right) \ldots \Omega\left(u_{n}\right)\right\rangle . \\
\mathcal{K}_{n} & :=\int_{0}^{t} \mathrm{~d} u_{1} \int_{0}^{u_{1}} \mathrm{~d} u_{2} \ldots \int_{0}^{u_{n-1}} \mathrm{~d} u_{n}\left\langle\Omega\left(u_{1}\right) \Omega\left(u_{2}\right) \ldots \Omega\left(u_{n}\right)\right\rangle_{c},
\end{aligned}
$$

respectively. It is worth stressing that respect to the "time" parameter $u$, the result of the ordering $\{\ldots\}_{O}$ associated to the $t$-ordered exponential, is different for the case of the $M$ respect to the case of the $M_{O}$ mappings. This is because these mappings act on different spaces. In fact, in the $\mathfrak{M}$ vector space of which the basis is given by the tensor products of $\Omega\left(u_{i}\right)$ (we recall that the time parameter $u$ does not uniquely identify the base element $\Omega$ ), $M=O$ leads to an ordering of the basis respect to the time $u$, e.g.,

$$
\left\{\Omega\left(u_{i}\right) \Omega\left(u_{j}\right)\right\}_{O}=\left\{\begin{array}{l}
\Omega\left(u_{i}\right) \Omega\left(u_{j}\right) \text { for } u_{i}>u_{j} \\
\Omega\left(u_{j}\right) \Omega\left(u_{i}\right) \text { for } u_{j}>u_{i} .
\end{array}\right.
$$

Therefore, in this space, each one of the elements identified by the parameter $u$ is $M$ unconnected to any other element identified by a paramter $u^{\prime} \neq u$. On the other hand, the mapping $M_{O}=O$, defined by assuming that $\exp _{M_{O}}[\ldots]$ of Eq. (39) is a $t$-ordered exponential, acts on the vector space with basis given by the tensor products of cumulants $\kappa_{n}^{(\tilde{M}, \tilde{O})}\left(u_{i_{1}}, u_{i_{2}}, \ldots, u_{i_{n}}\right):=\left\{\Omega\left(u_{1}\right) \Omega\left(u_{2}\right) \ldots \Omega\left(u_{n}\right)\right\}_{\tilde{M}_{c}}$ (see Remark $\mathbf{H}$ ) that depend on $n$ time parameters and where the ordering is respect to the largest time inside each cumulant, e.g. (assuming $u_{i_{1}}>u_{i_{k}}$ and $u_{j_{1}}>u_{j_{k}}, k>1$ ):

$$
\begin{aligned}
& \left\{\left\{\Omega\left(u_{i_{1}}\right) \Omega\left(u_{i_{2}}\right) \ldots \Omega\left(u_{i_{n}}\right)\right\}_{\tilde{M}_{c}} \otimes\left\{\Omega\left(u_{j_{1}}\right) \Omega\left(u_{j_{2}}\right) \ldots \Omega\left(u_{j_{m}}\right)\right\}_{\tilde{M}_{c}}\right\}_{O} \\
= & \left\{\begin{array}{l}
\left\{\Omega\left(u_{i_{1}}\right) \Omega\left(u_{i_{2}}\right) \ldots \Omega\left(u_{i_{n}}\right)\right\}_{\tilde{M}_{c}} \otimes\left\{\Omega\left(u_{j_{1}}\right) \Omega\left(u_{j_{2}}\right) \ldots \Omega\left(u_{j_{m}}\right)\right\}_{\tilde{M}_{c}} \text { for } u_{i_{1}}>u_{j_{1}} \\
\left\{\Omega\left(u_{j_{1}}\right) \Omega\left(u_{j_{2}}\right) \ldots \Omega\left(u_{j_{m}}\right)\right\}_{\tilde{M}_{c}} \otimes\left\{\Omega\left(u_{i_{1}}\right) \Omega\left(u_{i_{2}}\right) \ldots \Omega\left(u_{i_{n}}\right)\right\}_{\tilde{M}_{c}} \text { for } u_{j_{1}}>u_{i_{1}},
\end{array}\right.
\end{aligned}
$$

no matter the relationship among the other times. Therefore $M_{O}=O$ gives a partial ordering respect to the time $u_{i}$ (see the examples in Section IVD).

\section{B. The fundamental property for cumulants}

Within the framework defined in the previous sections, the approach to generalized cumulants results well-founded because, as it is shown hereafter, it follows that a cumulant 
is vanishing if it refers to $\mathfrak{u}$-independent stochastic processes (or, that is the same, to $M$ unconnected sets). In this respect, the following Lemma is central for the present work and, after Definition H, Lemma 1 and Theorem 2, it goes without the need of demonstration:

Lemma 2. For any $u \in \mathcal{T} \subset \mathcal{U}$ and $u^{\prime} \in \mathcal{T}^{\prime} \subset \mathcal{U}$ two stochastic processes $\Omega(u)$ and $\Omega\left(u^{\prime}\right)$ are reciprocally $\mathfrak{u}$-independent if and only if we have

$$
\exp _{M_{O}}[\mathcal{K}(\mathcal{T})] \exp _{M_{O}}\left[\mathcal{K}\left(\mathcal{T}^{\prime}\right)\right]=\exp _{M_{O}}\left[\mathcal{K}(\mathcal{T})+\mathcal{K}\left(\mathcal{T}^{\prime}\right)\right]
$$

Remark I. By Theorem [ , from Eq. (52) it follows that for any $u \in \mathcal{T} \subset \mathcal{U}$ and $u^{\prime} \in \mathcal{T}^{\prime} \subset$ $\mathcal{U}$ we have that $\mathcal{K}(u)$ and $\mathcal{K}\left(u^{\prime}\right)$ are $M$-unconnected to each other. Thus, we can restate Lemma 0 as follows: using the $M_{O}$ map to define the generalized cumulant generating function leads to a bijective mapping between couples of reciprocally $M$-unconnected sets in the $\mathfrak{M}$ space of the stochastic process $\Omega(u)$ and couples of reciprocally $M$-unconnected sets in the $\mathfrak{M}$ space of the generalized cumulant generating function $\mathcal{K}(u)$.

Definition I. The fundamental property of cumulants is the statement that in the same

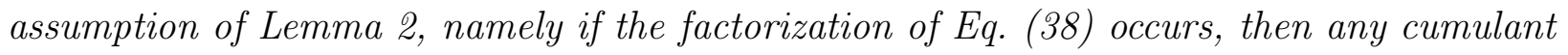
mixing the average of the $\Omega$ random operators of the two sets $\mathcal{T}$ and $\mathcal{T}^{\prime}$ is vanishing.

Lemma 3. Under the same assumption of Lemma圈, the fundamental property of cumulants holds.

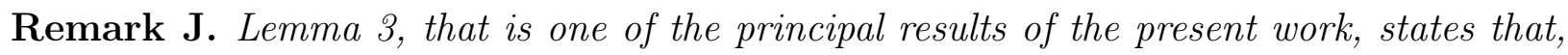
although for non-commuting processes Eq. (52), in general, does not hold, it automatically holds when the stochastic processes are $\mathfrak{u}$-independent according to Definition $\mathbb{H}$.

Remark K. Usually, the definition of the $\mathfrak{M}$ space of the stochastic process $\Omega(u)$, the introduction of the generalized moment generating function and of the related $M$-projection map, cam naturally from the "starting facts" of the specific real problem (e.g. from physics) we are interested in, while cumulants are introduced by hand for convenience in different ways (provided Eq. 44) is satisfied). One might wonder why for cumulants we have restrict the choice of the M-projection map to the less general M-ordering type. This is because thanks to this constraint Eq. (52) is automatically fulfilled for independent stochastic processes $\Omega(u)$ and $\Omega\left(u^{\prime}\right)$. This is not always the case for cumulants defined by using the a M-projection map without restrictions (it should be checked case by case) 
After Lemma 2 the demonstration of Lemma 3 is trivial being the same holding for standard c-number stochastic processes: comparing Eq. (39) with Eq. (38) we see that Eq. (52) means that $\mathcal{K}\left(\mathcal{T} \cup \mathcal{T}^{\prime}\right)=\mathcal{K}(\mathcal{T})+\mathcal{K}\left(\mathcal{T}^{\prime}\right)$, thus $\mathcal{K}\left(\mathcal{T} \cup \mathcal{T}^{\prime}\right)$ does not have terms mixing the averages of the $\Omega$ stochastic operators of the two sets $\mathcal{T}$ and $\mathcal{T}^{\prime}$.


by Theorem 2, is strictly related to the factorization of the exponential of sums (and, in turn, to the Definition [H of $\mathfrak{u}$-unconnected stochastic processes). Shortly (in the frame of the present formal approach) the fundamental property of cumulants holds if the "statistical" independence is defined by the factorization of the averages given in Eq. (38). The lack of clarity about the definition of statistical independence for q-numbers has been the main source of confusion in the debate about the validity of the Kubo's approach to generalized cumulants. In fact, in his seminal work 1 Kubo enunciated his Theorem I in the following way: ".. a cumulant is zero if the elements are divided into two or more groups which are statistically independent." And, in the following Corollary: "A cumulant is zero if one of the variables in it is independent of the others. Conversely, a cumulant is not zero if and only if the variables in it are statistically connected." Both the theorems and the corollary are true for commuting random processes. For q-numbers they are also true only if we identify the statistical independence with the factorization of the moment generator function as in Definition G. The extension to q-number stochastic processes leads also to the introduction of the $\mathfrak{u}$-independence as in Definition $[\mathrm{H}$. In our opinion Fox misunderstood this point, because his "counterexample", that (for him) should invalidate the Kubo's theorems, simply is an archetypal case where the statistical independence of the stochastic coefficients (c-numbers) of an operator is not enough to guarantee the factorization of the moment generating function.

Remark M. Another point of the Kubo's work that has originated some confusion is the not so clear definition of the $M$ maps. In particular, it has not be clarified which are the domain spaces where these maps are defined. As we have shown in an example here above (see Eqs. (47)-(51)), the $M=0$ map (t-ordering), acts in different ways in different $\mathfrak{M}$ vector spaces (see e.g.,,$^{10}$, where Eqs. (2.14a)-(2.14d) are not correct because of a not proper definition of the $M_{O}=O$ operator).

Before to pass to the second formal part of the present work, concerning the explicit relationship between generalized moments and generalized cumulants, we discuss a little 
about the above results. Although formally it sounds well, one could (and should) wonder if, in any "realistic" model of some interest, the assumption of Lemma 2 (factorization of the generalized moment generating function) is fulfilled and, if it is the case, if it has a clear physical (or whatever interesting) interpretation. There are cases in physics where this happens. A class of cases it that treated in Section IVD1, where the generalized moments are reduced density matrices and they naturally factorize for non-interacting particles (or, asymptotically, for parts of a very large system). Another situation, probably the most common in Physics, it that for which the $M$ mapping is the time ordering: $M=O$ (also treated in detail in Sections IVD 2, IVD 3). In this case, as already remarked after Eq. (50), each $\Omega(u) \in \mathfrak{B}$ is $M$-unconnected to any other element of $\mathfrak{B}$, with a different value of the parameter $u$. It follows that in $\mathfrak{B}$, the $M=O$ map defines an ordered partition of $M$-unconnected subsets $\{\Omega(u)\}$, each one associated with a different value of the "time" parameter $u$ : in the space $\mathfrak{M}$ the $M=O$ map defines subsets with different "time-scales" that are $M$-unconnected to each other. More precisely, for any couple of subsets $\mathcal{T}, \mathcal{T}^{\prime}$ of $\mathcal{U}$ such that $\forall u \in \mathcal{T}, u^{\prime} \in \mathcal{T}^{\prime}$, we have $u>u^{\prime}$, then $\left\{\Omega\left(u_{1}\right) \Omega\left(u_{2}\right) \ldots \Omega\left(u_{n}\right) \Omega\left(u_{1}^{\prime}\right) \Omega\left(u_{2}^{\prime}\right) \ldots \Omega\left(u_{m}^{\prime}\right)\right\}_{O}=$ $\left\{\Omega\left(u_{1}\right) \Omega\left(u_{2}\right) \ldots \Omega\left(u_{n}\right)\right\}_{O}\left\{\Omega\left(u_{1}^{\prime}\right) \Omega\left(u_{2}^{\prime}\right) \ldots \Omega\left(u_{m}^{\prime}\right)\right\}_{O}$. Now, if the average process $\langle\ldots\rangle$ is done by using a measure that factorizes in two separate measures for the two subsets with different "time-scales" $\mathcal{T}$ and $\mathcal{T}$ ', then, the same two subsets of $\mathfrak{M}$ are $M$-unconnected respect to the composite map $\tilde{M}=\langle.\rangle \circ M$. Thus, by Theorem 2 and Definition $\mathrm{H}$, it follows that the factorization of Eq. 38 is satisfied.

Let us see that explicitly. We assume that $\Omega(t)=\mathcal{L}(t) \xi(t)$, where $\mathcal{L}(t)$ is some timedependent operator (e.g., a matrix, a differential operator, a Lie-adjoint operator etc.), that does not commute with itself for different times: $\left[\mathcal{L}\left(t_{1}\right), \mathcal{L}\left(t_{2}\right)\right] \neq 0$ and $\xi(t)$ is some cnumber stochastic process. We indicate with $p\left(\xi_{1}, t_{1} ; \xi_{2}, t_{2} ; \ldots ; \xi_{n}, t: n\right)$ the joint probability density function to have (with some abuse of notation) $\xi\left(t_{1}\right)=\xi_{1}$ and $\xi\left(t_{2}\right)=\xi_{2}$ and...and $\xi\left(t_{n}\right)=\xi_{n}$. Let us assume now that there is a time $\bar{t}$ such that the instances of $\xi(t)$ at times $t>\bar{t}$ are statistically uncorrelated with the instances of $\xi(t)$ at times $t<\bar{t}$. Thus, if $t_{1}>\bar{t}$ and $t_{2}<\bar{t}$ we have $p\left(\xi_{1}, t_{1} ; \xi_{2}, t_{2}\right)=p\left(\xi_{1}, t_{1}\right) p\left(\xi_{2}, t_{2}\right)$. Then, the generalized moment 
generating function for the stochastic operator $\Omega\left(t_{1}\right), \Omega\left(t_{2}\right)$ is

$$
\begin{aligned}
\left\langle\left\{e^{\mathcal{L}\left(t_{1}\right) \xi\left(t_{1}\right) \mathrm{d} t+\mathcal{L}\left(t_{2}\right) \xi\left(t_{2}\right) \mathrm{d} t}\right\}_{O}\right\rangle & :=\int \mathrm{d} \xi_{1} \mathrm{~d} \xi_{2} p\left(\xi_{1}, t_{1} ; \xi_{2}, t_{2}\right)\left\{e^{\mathcal{L}\left(t_{1}\right) \xi\left(t_{1}\right) \mathrm{d} t+\mathcal{L}\left(t_{2}\right) \xi\left(t_{2}\right) \mathrm{d} t}\right\}_{O} \\
& =\left\{\int \mathrm{d} \xi_{1} \mathrm{~d} \xi_{2} p\left(\xi_{1}, t_{1}\right) p\left(\xi_{2}, t_{2}\right) e^{\mathcal{L}\left(t_{1}\right) \xi\left(t_{1}\right) \mathrm{d} t+\mathcal{L}\left(t_{2}\right) \xi\left(t_{2}\right) \mathrm{d} t}\right\}_{O} \\
& =\left\langle e^{\mathcal{L}\left(t_{1}\right) \xi\left(t_{1}\right) \mathrm{d} t}\right\rangle\left\langle e^{\mathcal{L}\left(t_{2}\right) \xi\left(t_{2}\right) \mathrm{d} t}\right\rangle
\end{aligned}
$$

And, for $t>\bar{t}$, we also have

$$
\left\langle\exp _{O}\left[\int_{0}^{t} \mathcal{L}(u) \xi(u) \mathrm{d} u\right]\right\rangle:=\lim _{\epsilon \rightarrow 0}\left\langle\exp _{O}\left[\int_{\bar{t}+\epsilon}^{t} \mathcal{L}(u) \xi(u) \mathrm{d} u\right]\right\rangle\left\langle\exp _{O}\left[\int_{0}^{\bar{t}-\epsilon} \mathcal{L}(u) \xi(u) \mathrm{d} u\right]\right\rangle
$$

\section{A closed-form formula for cumulants}

\section{Background}

As it is well known, for commuting stochastic processes a closed-form formula was worked out by Meeron ${ }^{43}$ (Appendix). In Kubo's basic paper ${ }^{1}$, a similar formula has been proposed also for non-commuting processes. That was strongly criticized by Fox ${ }^{6}$ for the case of timeordered cumulants. In a subsequent paper, Apresyan $\stackrel{44}{ }$, supporting Kubo's work, proposed a Meeron-Kubo's equivalent formula, that, in turn, Fox ${ }^{7}$ criticized again, presenting what he purports to be a counterexample. As we have already observed in Remark $\mathrm{L}$, in the Fox criticism there was confusion (actually due to some mistake in the original Kubo's paper) generated by the identification of the partition defined by unconnected sets and the partition given by the sets of statistically independent stochastic operators. Moreover, more confusion, supporting the Fox arguments, was introduced by Fox himself ${ }^{45}$ and by other authors (e.g.,$\left.\frac{10}{}\right)$, which made a too generalized use of the Meeron-Kubo formula for cumulants.

In this section, we shall show that using the definitions of cumulants given in Eq. (39), a Meeron formula holds, but only for the expression the gives the operator moments in terms of operator cumulants. A general inverse formula (namely, valid for any $M$-projection mapping), that gives generalized cumulants in terms of generalized moments, does not hold true for operators (or, if it exists, is not so straightforward). Actually, in some cases of

physical interest, it is possible to find such a closed-form formula (see the examples in Section IVD). Thus Fox, despite that he was wrong when stated $\underline{\underline{6}}$ that Kubo's approach to 
generalized cumulants does not lead to the validity of the fundamental property of cumulants, he was partially right affirming that the Meeron-Kubo's closed-form formula cannot be applied.

\section{Main results}

We start inserting in Eq. (39) the series $\mathcal{M}=\sum_{n=0}^{\infty} \mathcal{M}_{n}$ and $\mathcal{K}=\sum_{n=1}^{\infty} \mathcal{K}_{n}$ :

$$
\sum_{m=0}^{\infty} \frac{1}{m !}\left\{\left(\sum_{r=1}^{\infty} \mathcal{K}_{r}\right)^{m}\right\}_{M_{O}}=\sum_{n=0}^{\infty} \mathcal{M}_{n}
$$

The Meeron closed-form formula that gives the generalized moment generating components $\mathcal{M}_{n}$ in terms of sums of products of generalized cumulant generating components $\mathcal{K}_{r}, r \leq n$, is obtained by rearranging the sums in the 1.h.s. of Eq. (55). By Definition A, point (ii), the $M$-projection map does not interfere with this kind of arrangement of the addends of the sums. Thus, the formula is the same as that of Meeron ${ }^{43}$ (Eq. (A7)), apart the M-projection mapping applied to each addend of the sums:

Proposition 2. The generalized moment generating component of order $n$ is given in terms of the generalized cumulant generating components of order $r \leq n$ by the following expression:

$$
\mathcal{M}_{n}=\sum_{\text {Part. of } n}\left\{\prod_{r=1}^{n} \frac{1}{s_{r} !}\left(\mathcal{K}_{r}\right)^{s_{r}}\right\}_{M_{O}}
$$

in which the sum is over all the possible ways to partition $n$ "elements" such that: one partition is made of $s_{1}=n$ groups with $r=1$ element, another partition is made of only one group $\left(s_{n}=1\right)$ with $r=n$ elements, a generic partition is made of $s_{1}$ groups with $r=1$ elements and $s_{2}$ groups with $r=2$ elements and etc..., such that $\sum_{r=1}^{n} r s_{r}=n$.

We report shortly the demonstration in Appendix B.

For what concerns the inverse Meeron-like formula:

$$
\mathcal{K}_{n}=\sum_{\text {Part.of } n}(-1)^{p-1}(p-1) ! \prod_{r=1}^{n} \frac{1}{s_{r} !}\left(\mathcal{M}_{r}\right)^{s_{r}}, \quad p=\sum_{r=1}^{n} s_{r}, \quad \mathcal{M}_{r} \text { are c-numbers }
$$

it cannot be generalized to q-number stochastic processes just by applying one of the $M$-projection mappings $\left(M_{O}\right.$ and/or $\left.M\right)$ to the addends. Actually, for non-commuting stochastic processes we are not able to find such a general formula. The reason is the 
following: for standard commuting stochastic processes to obtain such a result we start from Eq. (39) and, to get rid of the exponential in the l.h.s., we take the logarithm of both sides of the equation. Then, in the r.h.s. of the resulting equation, we expand this logarithm in power series of its argument. For non-commuting processes in the 1.h.s. of Eq. (39) we have a $M$-exponential instead of a standard exponential, and, because it is a projection map, it is not usually invertible. In conclusion, we guess that there is not a general definition for a generalized logarithm that corresponds to the inverse of the $M$-exponential (however, we do hope that we will be proved wrong).

In our opinion this is the real mistake in the Kubo's paper ${ }^{1}$ that has originated the Fox criticism and that, to the best of our knowledge, has not been cured so far. Case by case, the formula that gives directly generalized cumulants from generalized moments can be found, as it will be shown in Section IVD. A way to face the problem (still case by case) is to consider the relation between cumulants and moments in the frame of a combinatoric calculus on partition lattices. By expressing in terms of set-partitions of $n$ distinguishable objects the formula that gives the $n$-moment in terms of cumulants (see Eq. ([62)), and taking into account the specific $M$ and $M_{O}$ maps that define the rule for managing (e.g., by ordering) the elements in the partitions, then, at least in principle, the Möbius inversion result on the partition lattice should lead to the inverse formula ${ }^{46}$ for the specific case of interest.

Also when this formula is known, often the easier practical way to get $\mathcal{K}_{n}$ from $\mathcal{M}_{r}$ is to iteratively use Eq. (56). We explicitly do that by running some iterations: we start with $\mathcal{K}_{1}=\mathcal{M}_{1}$ and

$$
\mathcal{K}_{2}=\mathcal{M}_{2}-\frac{1}{2}\left\{\left(\mathcal{K}_{1}\right)^{2}\right\}_{M_{O}}
$$

in which $\mathcal{K}_{1}=\mathcal{M}_{1}$,

$$
\mathcal{K}_{3}=\mathcal{M}_{3}-\left\{\mathcal{K}_{1} \otimes \mathcal{K}_{2}\right\}_{M_{O}}-\frac{1}{3 !}\left\{\left(\mathcal{K}_{1}\right)^{3}\right\}_{M_{O}}
$$

in which $\mathcal{K}_{1}=\mathcal{M}_{1}$ and $\mathcal{K}_{2}$ is obtained by Eq. (58),

$$
\mathcal{K}_{4}=\mathcal{M}_{4}-\frac{1}{2}\left\{\left(\mathcal{K}_{2}\right)^{2}\right\}_{M_{O}}-\frac{1}{2}\left\{\left(\mathcal{K}_{1}\right)^{2} \otimes \mathcal{K}_{2}\right\}_{M_{O}}-\frac{1}{4 !}\left\{\left(\mathcal{K}_{1}\right)^{4}\right\}_{M_{O}}
$$

in which $\mathcal{K}_{1}=\mathcal{M}_{1}$ and $\mathcal{K}_{2}$ and $\mathcal{K}_{3}$ are derived from Eq. (58) and Eq. (59), respectively,

$$
\begin{aligned}
\mathcal{K}_{5} & =\mathcal{M}_{5}-\left\{\mathcal{K}_{2} \otimes \mathcal{K}_{3}\right\}_{M_{O}}-\frac{1}{2}\left\{\mathcal{K}_{2}^{2} \otimes \mathcal{K}_{1}\right\}_{M_{O}} \\
& -\left\{\mathcal{K}_{1} \otimes \mathcal{K}_{4}\right\}_{M_{O}}-\frac{1}{2}\left\{\mathcal{K}_{1}^{2} \otimes \mathcal{K}_{3}\right\}_{M_{O}}-\frac{1}{3 !}\left\{\mathcal{K}_{1}^{3} \otimes \mathcal{K}_{2}\right\}_{M_{O}}-\frac{1}{5 !}\left\{\mathcal{K}_{1}^{5}\right\}_{M_{O}}
\end{aligned}
$$


where $\mathcal{K}_{1}=\mathcal{M}_{1}$ and $\mathcal{K}_{2}, \mathcal{K}_{3}$ and $\mathcal{K}_{4}$ are derived from Eq. (58), Eq. (59) and Eq. (60), respectively; and so on. Notice that if $\mathcal{M}_{1}=0$ then $\mathcal{K}_{2}=\mathcal{M}_{2}$ and $\mathcal{K}_{3}=\mathcal{M}_{3}$, whatever the rule $M_{O}$. To go ahead with the above iterative procedure we have to bear in mind how the $M_{O}$ map acts on the space of tensor products of the cumulant density functions of Eq. (43) (on which it is defined).

The same arguments that lead to Proposition 2 can also be used to write the analog closed-form formula that gives directly density moments in terms of density cumulants:

Lemma 4. The generalized n-moment density function $\mu_{n}^{(\tilde{M})}\left(u_{1}, u_{2}, \ldots u_{n}\right)$ is given in terms of the generalized cumulant density functions $\kappa_{n}^{\left(\tilde{M}, M_{O}\right)}\left(u_{1}, u_{2}, \ldots u_{m}\right)$ by the standard Meeron formula, taking care to apply the M-ordering map to the result:

$$
\mu_{n}^{(\tilde{M})}\left(u_{1}, u_{2}, \ldots u_{n}\right)=\sum_{p=1}^{n}\left(\sum_{\sum_{i=1}^{p} m_{i}=n}\left\{\prod_{i=1}^{p} \kappa_{m_{i}}^{\left(\tilde{M}, M_{O}\right)}\right\}_{M_{O}}\right)
$$

where $\kappa_{m_{i}}^{\left(\tilde{M}, M_{O}\right)}=\left\{\Omega\left(u_{j_{1}}, \Omega\left(u_{j_{2}}\right), \ldots, \Omega\left(u_{j_{m_{i}}}\right)\right\}_{\tilde{M}_{c}}\right.$ is the generalized $m_{i}$-cumulant density function, and $\left\{m_{i}\right\}$ is a subset of $n:=[1,2, \ldots, n]$, with $m_{i}$ elements.

An equivalent but more compact way to write Eq. (62) is the following:

$$
\left\{\Omega\left(u_{1}\right), \Omega\left(u_{2}\right), \ldots, \Omega\left(u_{n}\right)\right\}_{\tilde{M}}=\sum_{\pi(n)}\left\{\prod_{B \in \pi(n)}\left\{\prod_{i \in B} \Omega\left(u_{i}\right)\right\}_{\tilde{M}_{c}}\right\}_{M_{O}}
$$

where $\pi(n)$ runs through the list of al set-partitions (or grouping) of $n$ distinguishable objects and $B$ runs through the list of all blocks of the partition $\pi(n)$.

Remark N. The formal algebraic expression of Eq. (62) that gives generalized moments in terms of generalized cumulants does not depend on the nature of the generalized moments (that is related to the $\tilde{M}$ map), but it depends on the choice of the $M_{O}$ map. Of course, the choice of the $M_{O}$ map is not completely independent of $\tilde{M}$ but it is constrained by Eq. (44).

From a practical point of view, it is more convenient to rephrase the above lemma by adapting to the present general case the recipe of Roednik"47 (pag.27, "reverse transformation") (the Roednik-van Kampen analytical work on the operator-cumulants defined by the $t$-ordered exponential can be considered as an anticipation of the modern combinatorial approach to this subject): 
(i) Write a sequence of $n$-dots.

(ii) Partition them into non crossing subsequences $\{\ldots \ldots\}_{\tilde{M}_{c}}$ (cumulant averages inside of which the $M$ mapping is applied) by inserting these brackets in all possible ways (excluding empty subsequences).

(iii) For each partition write 1 on the first dot, and any permutation of the numerals $2,3, \ldots . ., n$ on the remaining dots, subject to the condition that subsequences with different ordering of the same numerals are considered equivalent (or, that is the same, take just one kind of ordering, for example, not decreasing).

(iv) Replace each numeral " $i$ " by " $\Omega\left(u_{i}\right)$ ".

(v) Apply the $M$-ordering map (the $M_{O}$ map, shortly) to each addend so obtained.

Here we explicitly write down the first four generalized density moments in terms of generalized density cumulants (notice, $\langle\Omega(u)\rangle=\langle\Omega(u)\rangle_{c}$ whatever the $M_{O}$ and $M$ mappings, we also shall use the shorthand notation $\Omega\left(u_{i}\right) \rightarrow i$ and $\Omega\left(u_{i}\right) \Omega\left(u_{j}\right) \rightarrow i \cdot j$, thus $\left\{i_{1} \cdot i_{2} \cdot \ldots \cdot i_{k}\right\}_{\tilde{M}_{c}}:=$ $\left.\left\langle\left\{i_{1} \cdot i_{2} \cdot \ldots \cdot i_{k}\right\}_{M}\right\rangle_{c}\right):$

$$
\begin{aligned}
\{i \cdot j\}_{\tilde{M}} & =\{i \cdot j\}_{\tilde{M}_{c}}+\{\langle i\rangle \otimes\langle j\rangle\}_{M_{O}} \\
\{i \cdot j \cdot r\}_{\tilde{M}} & =\{i \cdot j \cdot r\}_{\tilde{M}_{c}}+\{\langle i\rangle \otimes\langle j\rangle \otimes\langle r\rangle\}_{M_{O}} \\
& +\underbrace{\left\{\{i \cdot j\}_{\tilde{M}_{c}} \otimes\langle r\rangle\right\}_{M_{O}}+\left\{\{i \cdot r\}_{\tilde{M}_{c}} \otimes\langle j\rangle\right\}_{M_{O}}+\left\{\{j \cdot r\}_{\tilde{M}_{c}} \otimes\langle i\rangle\right\}_{M_{O}}}_{:=\left\{\{i \cdot j\}_{\tilde{M}_{c}} \otimes\langle r\rangle\right\}_{M_{O}}[3]} \\
\{i \cdot j \cdot r \cdot s\}_{\tilde{M}} & =\{i \cdot j \cdot r \cdot s\}_{\tilde{M}_{c}}+\{\langle i\rangle \otimes\langle j\rangle \otimes\langle r\rangle \otimes\langle s\rangle\}_{M_{O}} \\
& +\left\{\{i \cdot j \cdot r\}_{\tilde{M}_{c}} \otimes\langle s\rangle\right\}_{M_{O}}[4]+\left\{\{i \cdot j\}_{\tilde{M}_{c}} \otimes\{r \cdot s\}_{\tilde{M}_{c}}\right\}_{M_{O}}[3] \\
& +\left\{\{i \cdot j\}_{\tilde{M}_{c}} \otimes\langle r\rangle \otimes\langle s\rangle\right\}_{M_{O}}[6]
\end{aligned}
$$

Each number between square parentheses indicates a sum over distinct partitions having the same block sizes, so the fourth-order moment is, in general, a sum of 15 distinct density cumulant products. In some cases however the $M_{O}$ map could impose to discard some of terms we obtain in the r.h.s. of the Eq. (64). For example, in the case where $M_{O}=G$, illustrated in section IVD, we take only the partitions where the operators are totally ordered respect to the time parameter $u$, see Eq. (94). Another example is where the $M_{O \text {-projection }}$ includes a fully anti-symmetrizer operator (see Section IVD 1). In this case the relations 
of Eq. (64) have been extensively used to express reduced densities matrices of electrons in terms of cumulants that cancel quantities that are not size-extensive (see Eqs. (2.25)-(2.28) of the Ziesche seminal paper of ${ }^{13}$ and $\left.^{12,14-16,19}\right)$.

\section{A few important classes of cases}

In the most common cases $M=O$ (time ordering of the operators $\Omega(u)$ ) and we shall treat some of them in detail. However, before that, we want to discuss, albeit briefly, the application of our results to the context of quantum mechanics, and in particular to many body boson or fermion systems.

\section{Many identical particles}

For the sake of simplicity, we shall deal only with fermions, but the extension to bosons is straightforward. This is a field subjected to a very dynamic research activity, in particular concerning the reduced density matrix (RDM) approach and the related reduced density matrix cumulants (RDMC) technique, adopted by many research groups (e.g, $\underline{12-19,48,49}$ ). We think that the results of the present work can contribute to a simple systematic development of this research field. For the reader skilled in this matter, it should be enough to observe that the antisymmetric Grassmann (or exterior) product is commutative (when applied between tensors with the same number of upper and lower indices) and satisfy the conditions of Definition E for an $M$-ordering map. Thus, if we use it as the $M_{O}$ map introduced in Section IVA, we see that whatever the definition of the generating function for the RDM, Lemma 4, and the explicit expansion of Eq. (64) gives the same relation between RDM and RDMC we find in the specific literature (e.g., see TABLE II of ${ }^{15}$ ). Notice that in our treatment we don't need to introduce any additional Grassmann function/Schwinger probes.

Let us now give some more (few) details for not expert in this matter. Let us work in the frame of the second quantization, and let us define de $p$ particles RDM as

$$
\boldsymbol{D}_{\boldsymbol{p}}:=\boldsymbol{D}_{j_{1}, \ldots, j_{p}}^{i_{1}, \ldots, i_{p}}:=\frac{1}{p !}\left\langle\Psi_{N}\left|\hat{\boldsymbol{a}}_{i_{1}}^{\dagger} \cdots \hat{\boldsymbol{a}}_{i_{p}}^{\dagger} \hat{\boldsymbol{a}}_{j_{p}} \cdots \hat{\boldsymbol{a}}_{j_{1}}\right| \Psi_{N}\right\rangle
$$

where the number of indices implicitly specifies the tensor rank, a convention that shall be followed hereafter, $\Psi_{N}$ represent some state of the whole system of $N$ particles and, finally, 
as usual $\hat{\boldsymbol{a}}_{i_{p}}^{\dagger}\left(\hat{\boldsymbol{a}}_{j_{p}}\right)$ is the creation (annihilation) operator, for the quantum number $i_{p}\left(j_{p}\right)$. For example, if $\Psi_{N}$ is the pure state of a $N$-electrons system, the $N$-electron density matrix for the state $\Psi$ is the following projector:

$$
\boldsymbol{D}_{N}:=\left|\Psi_{N}\right\rangle\left\langle\Psi_{N}\right|=\frac{1}{N !}\left\langle\Psi_{N}\left|\hat{\boldsymbol{a}}_{i_{1}}^{\dagger} \cdots \hat{\boldsymbol{a}}_{i_{N}}^{\dagger} \hat{\boldsymbol{a}}_{j_{N}} \cdots \hat{\boldsymbol{a}}_{j_{1}}\right| \Psi_{N}\right\rangle
$$

of which a partial trace operation, applied to the indices from $p+1(p<N)$ to $N$ (by a factor $N ! / p !)$, gives, by definition, the $p$-RDM. An old result by Löwdin ${ }^{50}$ makes the RDM interesting: due to the antisymmetry of $\Psi, \boldsymbol{D}_{p}$ can be used to compute the exact expectation value of any $p$-electron operator that treats all $p$ electrons equivalently. Since electrons are indistinguishable according to the postulates of quantum mechanics, any valid observable must correspond to such an operator. Moreover, it is a consequence of empirical facts that all the $N$ particle operators used in quantum mechanics are the sum of one or two pairs operators. We shall not go deeper in describing the advantages of the RDM approach to many-particle systems, the interested reader can consult some of the above-cited literature. Here we are focused on the possibility of decomposing the RDM in generalized cumulants. There are many reasons for which this decomposition is advantageous. We just cite the fact that in the thermodynamic limit $(N \rightarrow \infty)$ RDMs are not extensive quantities (RDMCs are), so they do not necessarily become additively separable in the limit of noninteracting subsystems (RDMs are multiplicatively separable rather than additively separable). This is easily seen: consider a composite system of identical particles made of two noninteracting subsystems, one with $p$ electrons (subsystem $A$ ) and the other with $q=N-p$ electrons (subsystem $B$ ). Thus the $\boldsymbol{D}_{2}$ RDM is given by $\boldsymbol{D}_{2}=\frac{1}{2}\left\langle\Psi_{A} \Psi_{B}\left|\hat{\boldsymbol{a}}_{i_{1}}^{\dagger} \hat{\boldsymbol{a}}_{i_{2}}^{\dagger} \hat{\boldsymbol{a}}_{j_{2}} \hat{\boldsymbol{a}}_{j_{1}}\right| \Psi_{A} \Psi_{B}\right\rangle$, and it is clear that its matrix elements scales as $N^{2}$. On the other hand, the second reduced density matrix cumulant (RDMC) must scale as $N$ because, from the fundamental property of cumulants, the RDMC of the whole system is given by the sum of the RDMC of the two noninteracting parts.

To introduce the RDMC in the treatment of the present work, we start observing that Eq. (65) is written taking care of the normal ordering for the products of creation (left position) and annihilation (right position) operators, and we associate to this rule the $M$ map defined in Section IVA. Moreover, the average process, which combined with $M$ defines the $\tilde{M}$ map, makes fully antisymmetric the RDM, respect to all the indices. Comparing 
Eq. (65) with Eqs. (35)-(37) it is clear that the RDM-generating function is given by

$$
\mathcal{M}:=\left\langle\Psi\left|\exp _{M}\left[\sum_{i_{p}} \sum_{j_{p}} \hat{\boldsymbol{a}}_{i_{p}}^{\dagger} \hat{\boldsymbol{a}}_{j_{p}}\right]\right| \Psi\right\rangle:=\exp _{\tilde{M}}\left[\sum_{i_{p}} \sum_{j_{p}} \hat{\boldsymbol{a}}_{i_{p}}^{\dagger} \hat{\boldsymbol{a}}_{j_{p}}\right] .
$$

Following the procedure of the present work, we write the RDMC as:

$$
\boldsymbol{\Delta}_{\boldsymbol{p}}:=\boldsymbol{\Delta}_{j_{1}, \ldots, j_{p}}^{i_{1}, \ldots, i_{p}}:=\frac{1}{p !}\left\langle\hat{\boldsymbol{a}}_{i_{1}}^{\dagger} \cdots \hat{\boldsymbol{a}}_{i_{p}}^{\dagger} \hat{\boldsymbol{a}}_{j_{p}} \cdots \hat{\boldsymbol{a}}_{j_{1}}\right\rangle_{c}:=\frac{1}{p !}\left\{\hat{\boldsymbol{a}}_{i_{1}}^{\dagger} \cdots \hat{\boldsymbol{a}}_{i_{p}}^{\dagger} \hat{\boldsymbol{a}}_{j_{p}} \cdots \hat{\boldsymbol{a}}_{j_{1}}\right\}_{\tilde{M}_{c}}
$$

Following the definition of Eq. (39), the RDMC-generating function in the present case can be written as:

$$
\exp _{M_{O}}[\mathcal{K}]:=\mathcal{M}:=\exp _{\tilde{M}}\left[\sum_{i_{p}} \sum_{j_{p}} \hat{\boldsymbol{a}}_{i_{p}}^{\dagger} \hat{\boldsymbol{a}}_{j_{p}}\right]
$$

For the $M_{O}$ map, from which depends the specific definition of the RDMC, we know that we have different possibilities, but Eq. (44) must be satisfied. Because the RDM are fully antisymmetric respect to all the indices, the natural choice for the $M_{O}$ map is the operator that fully antisymmetrizes the indices of tensors, namely the Grassmann product, that, for two tensors $\mathcal{A}_{\boldsymbol{p}}$ and $\mathcal{B}_{\boldsymbol{q}}$ is defined by:

$$
\mathcal{A}_{\boldsymbol{p}} \wedge \mathcal{B}_{\boldsymbol{q}}:=\mathcal{A}_{j_{1}, \ldots, j_{p}}^{i_{1}, \ldots, i_{p}} \wedge \mathcal{B}_{j_{1}, \ldots, j_{q}}^{i_{1}, \ldots, i_{q}}:=\frac{1}{[(p+q) !]^{2}} \sum_{\pi, \sigma} \pi \sigma \epsilon(\pi) \epsilon(\sigma) \mathcal{A}_{j_{1}, \ldots, j_{p}}^{i_{1}, \ldots, i_{p}} \mathcal{B}_{j_{1}, \ldots, j_{q}}^{i_{1}, \ldots, i_{q}}
$$

where $\pi$ represents all permutations of the upper indices and $\sigma$ represents all permutations of the lower indices; the function $\epsilon(\pi)$ gives +1 for even permutations and -1 for odd permutations. The Grassmann product is linear and commutative (when applied between tensors with the same number of upper and lower indices). Thus we make the following choice for $M_{O}$ :

$$
\left\{\Delta_{p} \Delta_{q} \Delta_{s} \ldots\right\}_{M_{O}}:=\Delta_{p} \wedge \Delta_{q} \wedge \Delta_{s} \wedge \ldots
$$

With this choice, by exploiting Lemma 4or Eq. (64) we get exactly the same relation between $\mathrm{RDM}$ and RDMC we can find in the specific literature (e.g. $\left.\underline{15}^{4} \underline{49}\right)$ :

$$
\begin{aligned}
& \boldsymbol{D}_{1}=\boldsymbol{\Delta}_{1} \\
& \boldsymbol{D}_{2}=\boldsymbol{\Delta}_{2}+\boldsymbol{\Delta}_{1} \wedge \boldsymbol{\Delta}_{1} \\
& \boldsymbol{D}_{3}=\boldsymbol{\Delta}_{3}+\left(\boldsymbol{\Delta}_{1}\right)^{\wedge 3}+3 \boldsymbol{\Delta}_{2} \wedge \boldsymbol{\Delta}_{1} \\
& \boldsymbol{D}_{4}=\boldsymbol{\Delta}_{4}+\left(\boldsymbol{\Delta}_{1}\right)^{\wedge 4}+6 \boldsymbol{\Delta}_{2} \wedge\left(\boldsymbol{\Delta}_{1}\right)^{\wedge 2}+4 \boldsymbol{\Delta}_{3} \wedge \boldsymbol{\Delta}_{1}+3\left(\boldsymbol{\Delta}_{2}\right)^{\wedge 2}
\end{aligned}
$$


where $\mathcal{A}^{\wedge n}:=\underbrace{\mathcal{A} \wedge \mathcal{A} \wedge \ldots \wedge \mathcal{A}}_{n \text { times }}$. Notice that in the expression for $\boldsymbol{D}_{2}$ in the r.h.s. of Eq. (71), the term $\boldsymbol{\Delta}_{1} \wedge \boldsymbol{\Delta}_{1}$ is the one that for $N \rightarrow \infty$ scales with $N^{2}$, while the second RDMC represents the correlations, that scales with $N$, because for uncorrelated particles $\boldsymbol{\Delta}_{2}$ is additive.

In the same way, we can treat the $p$-density operators (or the $p$-density functions) or the Green's functions of electrons, which are the time-dependent version (in Heisenberg picture) of the $\mathrm{RDMs}^{13}$.

\section{The classical time ordered cumulants}

In statistical mechanics, both concerning the stochastic Langevin equation (see Eqs. (10)(8)) or the treatment of spin systems ${ }^{10}$, the case, $M=O$ (chronological ordering of the original basis constituted by the operators $\Omega(u)$ ) is dominant. In this case the formal expression of the generalized moment and cumulant density functions are given in Eq. (47), and, from the cumulant side, the original basis is given by the generalized cumulant density functions:

$$
\kappa_{n}^{\left(\tilde{O}, M_{O}\right)}\left(u_{1}, u_{2}, \ldots, u_{n}\right)=\left\langle\left\{\Omega\left(u_{1}\right) \Omega\left(u_{2}\right) \ldots \Omega\left(u_{n}\right)\right\}_{O}\right\rangle_{c} .
$$

In terms of this basis, the formal expression for the cumulant generating components are (see Eq. (49)):

$$
\begin{aligned}
& \mathcal{K}(t)=1+\int_{0}^{t} \mathrm{~d} u\langle\Omega(u)\rangle+\int_{0}^{t} \mathrm{~d} u_{1} \int_{0}^{u_{1}} \mathrm{~d} u_{2}\left\langle\Omega\left(u_{1}\right) \Omega\left(u_{2}\right)\right\rangle_{c} \\
& +\ldots+\int_{0}^{t} \mathrm{~d} u_{1} \int_{0}^{u_{1}} \mathrm{~d} u_{2} \ldots \int_{0}^{u_{n-1}} \mathrm{~d} u_{n}\left\langle\Omega\left(u_{1}\right) \Omega\left(u_{2}\right) \ldots \Omega\left(u_{n}\right)\right\rangle_{c}+\ldots
\end{aligned}
$$

Eq. (63) in this case becomes:

$$
\left\langle\left\{\Omega\left(u_{1}\right), \Omega\left(u_{2}\right), \ldots, \Omega\left(u_{n}\right)\right\}_{O}\right\rangle=\sum_{\pi(n)}\left\{\prod_{B \in \pi(n)}\left\langle\left\{\prod_{i \in B} \Omega\left(u_{i}\right)\right\}_{O}\right\rangle_{c}\right\}_{M_{O}} .
$$

Once we have set $M=O$, the relationship between cumulants and moments depends on the specific $M_{O}$ map we choose. In fact for $M=O$, the constraint in Eqs. (44)-(45) leaves some

freedom. This is made clear by using the following argument: $M=O$ means "chronological ordering" of the elements of the original basis given by the stochastic operators $\Omega(u)$. Because 
these operators depend only on one time parameter, this ordering is well defined. However, from the cumulant side, the elements of the original basis depend on many time parameters (see Eq. (172)), thus we have to specify (or choose) which of them is involved in the ordering process associated to the $M_{O}$ map. Two different choices are here presented, the first one in the present Section, the second one in the next Section.

If we know (or if we want) that the generalized moment generating function satisfies a local time differential equation, like

$$
\partial_{t} \mathcal{M}=\mathfrak{F}(t) \mathcal{M}
$$

we have

$$
\mathcal{M}(t)=\overleftarrow{\exp }\left[\int_{0}^{t} \mathrm{~d} u \mathfrak{F}(u)\right]
$$

thus,

$$
\mathcal{M}(t)=\overleftarrow{\exp }\left[\int_{0}^{t} \mathrm{~d} u \mathfrak{F}(u)\right]:=\exp _{M_{O}}[\mathcal{K}]
$$

From Eq. (77) it follows that $\mathfrak{F}(t)=\left(\partial_{t} \mathcal{K}(t)\right)$, namely

$$
\begin{aligned}
\mathcal{M}(t) & =\exp _{M_{O}}[\mathcal{K}]=\overleftarrow{\exp }\left[\int_{0}^{t} \mathrm{~d} u\left(\partial_{u} \mathcal{K}(u)\right)\right] \\
& =1+\int_{0}^{t} \mathrm{~d} u_{1}\left(\partial_{u_{1}} \mathcal{K}\left(u_{1}\right)\right)+\int_{0}^{t} \mathrm{~d} u_{1} \int_{0}^{u_{1}} \mathrm{~d} u_{2}\left(\partial_{u_{1}} \mathcal{K}\left(u_{1}\right)\right) \otimes\left(\partial_{u_{2}} \mathcal{K}\left(u_{2}\right)\right) \\
& +\int_{0}^{t} \mathrm{~d} u_{1} \int_{0}^{u_{1}} \mathrm{~d} u_{2} \int_{0}^{u_{2}} \mathrm{~d} u_{3}\left(\partial_{u_{1}} \mathcal{K}\left(u_{1}\right)\right) \otimes\left(\partial_{u_{2}} \mathcal{K}\left(u_{2}\right)\right) \otimes\left(\partial_{u_{3}} \mathcal{K}\left(u_{3}\right)\right)+\ldots
\end{aligned}
$$

Exploiting Eq. (73) it is easy to see that the series of Eq. (178) leads to choose $M_{O}=O$ defined in this way: in any tensor product $\left(u_{i} \geq u_{j}\right.$ if $\left.j \geq i\right)$ such as

$$
. . \otimes\left\langle\Omega\left(u_{i}\right) \Omega\left(u_{i+1}\right) \ldots \Omega\left(u_{i+n-1}\right)\right\rangle_{c} \otimes\left\langle\Omega\left(u_{j}\right) \Omega\left(u_{j+1}\right) \ldots \Omega\left(u_{i+m-1}\right)\right\rangle_{c} \otimes \ldots
$$

the $M_{O}$ map imposes a decreasing time ordering from left to right between the first parameter $u_{i}$ of any cumulant density function and the first parameter $u_{j}$ of the next cumulant density function, namely, $u_{i} \geq u_{j}$. This kind of ordering for the product of cumulant density functions is called Partial Time Ordering (PTO). Using this rule and reminding that the $M=O$ mapping imposes a time ordering inside any average, by exploiting Eq. (64) $\left(u_{i} \geq u_{j}\right.$ 
if $j \geq i$ ) it is easy to write the first four density moments in terms of cumulants:

$$
\begin{aligned}
\langle 1 \cdot 2\rangle & =\langle 1 \cdot 2\rangle_{c}+\langle 1\rangle \otimes\langle 2\rangle \\
\langle 1 \cdot 2 \cdot 3\rangle & =\langle 1 \cdot 2 \cdot 3\rangle_{c}+\langle 1\rangle \otimes\langle 2\rangle \otimes\langle 3\rangle \\
{[3 \text { terms: }] } & +\langle 1 \cdot 2\rangle_{c} \otimes\langle 3\rangle+\langle 1 \cdot 3\rangle_{c} \otimes\langle 2\rangle+\langle 1\rangle \otimes\langle 2 \cdot 3\rangle_{c} \\
\langle 1 \cdot 2 \cdot 3 \cdot 4\rangle & =\langle 1 \cdot 2 \cdot 3 \cdot 4\rangle_{c}+\langle 1\rangle \otimes\langle 2\rangle \otimes\langle 3\rangle \otimes\langle 4\rangle \\
{[4 \text { terms: }] } & +\langle 1 \cdot 2 \cdot 3\rangle_{c} \otimes\langle 4\rangle+\langle 1\rangle \otimes\langle 2 \cdot 3 \cdot 4\rangle_{c}+\langle 1 \cdot 3 \cdot 4\rangle_{c} \otimes\langle 2\rangle+\langle 1 \cdot 2 \cdot 4\rangle_{c} \otimes\langle 3\rangle \\
{[3 \text { terms: }] } & +\langle 1 \cdot 2\rangle_{c} \otimes\langle 3 \cdot 4\rangle_{c}+\langle 1 \cdot 3\rangle_{c} \otimes\langle 2 \cdot 4\rangle_{c}+\langle 1 \cdot 4\rangle_{c} \otimes\langle 2 \cdot 3\rangle_{c} \\
{[6 \text { terms: }] } & +\langle 1 \cdot 2\rangle_{c} \otimes\langle 3\rangle \otimes\langle 4\rangle+\langle 1 \cdot 3\rangle_{c} \otimes\langle 2\rangle \otimes\langle 4\rangle+\langle 1 \cdot 4\rangle_{c} \otimes\langle 2\rangle \otimes\langle 3\rangle \\
& +\langle 1\rangle \otimes\langle 2 \cdot 3\rangle_{c} \otimes\langle 4\rangle+\langle 1\rangle \otimes\langle 2\rangle \otimes\langle 3 \cdot 4\rangle_{c}+\langle 1\rangle \otimes\langle 2 \cdot 4\rangle_{c} \otimes\langle 3\rangle
\end{aligned}
$$

in which the partial ordering of the numerals is apparent. Eq. (179) is the same we have in the case of commuting stochastic processes, but here the terms are re-arranged following two rules (that are ineffective in the case of c-numbers):

- inside each group (or average) both in the 1.h.s. and in the r.h.s. of Eq. (79) it is important to keep the ordering of numerals, increasing from left to right (this is due to the $M=O$ map);

- in each addend, the ordering in the sequence of groups multiplication is such that the first numeral of any group is lesser than the first numeral of the next group (this is because of the $M_{O}$ mapping chosen for this case).

By using the combinatorial approach of set partitions, we can exploit the Möbius inversion formula (see for example ${ }^{9}$ ) to invert Eq. (63) and obtain:

$$
\left\langle\left\{\Omega\left(u_{1}\right), \Omega\left(u_{2}\right), \ldots, \Omega\left(u_{n}\right)\right\}_{O}\right\rangle_{c}=\sum_{\pi(n)}(-1)^{|\pi|-1} \sum_{P(B)} P \prod_{B \in \pi(n)}\left\langle\left\{\prod_{i \in B} \Omega\left(u_{i}\right)\right\}_{O}\right\rangle
$$

where, again, $\pi$ runs through the list of al set-partitions (or grouping) of $n$ distinguishable objects, $B$ runs through the list of all blocks of the partition $\pi,|\pi|$ is the number of blocks in the partition $\pi$ (the number of blocks, corresponding to $p$ in Eq. (62) ), $P$ is the permutation operator acting on the sequence of $|\pi|$ blocks $B$, but keeping fixed to the left side the one that contains (and then starts) with the numeral 1. Of course, the number of these permutations are $(|\pi|-1)$ ! We stress that, unlike Eq. (174), Eq. (180) is not the Meeron formula, modified 
by simply inserting some $M$-projection/ordering map. In fact here we have a sum over the permutations of blocks that is not present in the standard commuting processes case (where we have a factor $(|\pi|-1)$ ! in place of the same sum). An example may serve for illustration: from Eq. (80) we obtain, for the third cumulant density function, the following expression

$$
\begin{aligned}
\langle 1 \cdot 2 \cdot 3\rangle_{c}= & \langle 1 \cdot 2 \cdot 3\rangle-\langle 1 \cdot 2\rangle_{c} \otimes\langle 3\rangle-\langle 1 \cdot 3\rangle \otimes\langle 2\rangle-\langle 1\rangle \otimes\langle 2 \cdot 3\rangle \\
& +\langle 1\rangle \otimes\langle 2\rangle \otimes\langle 3\rangle+\langle 1\rangle \otimes\langle 3\rangle \otimes\langle 2\rangle ;
\end{aligned}
$$

the last two addends came out from the sum of the permutations of the two last groups (made of just one element, 2 or 3). In the case of c-number stochastic processes (and, unfortunately, also in the wrong closed-form formula, Eq. (6.9) of Kubo $\frac{1}{}$ ) we can collect them in only one addend with a factor $(3-1) !=2$.

Eq. (80) is a compact way to express the procedure found by van Kampen ${ }^{21}, 22$ and then Roednik ${ }^{47}$ (pag.27) by using a direct calculus approach. At the end of the next section we shall reformulate this result in a slightly different, but equivalent, way.

\section{Green operator and totally ordered cumulants}

Again with $M=O$, let assume now that the generalized moment generating function, instead of satisfying a local equation of motion as (75), satisfies an integral (or master) equation:

$$
\partial_{t} \mathcal{M}=\int_{0}^{t} \mathrm{~d} u G(t, u) \otimes \mathcal{M}(u) .
$$

This equation is, of course, very common in physics (and not only in physics). For example, it is obtained by using a Zwanzig projection operator technique (e.g. $\underline{23}, \underline{51} \underline{\underline{53}}$ ). Concerning this case, note that while Terwiel ${ }^{23}$ proved a cluster property for the "partial kernels" obtained using the Zwanzig projection approach, for Fox ${ }^{\underline{6}}$ "his proof does not establish directly the factorization property for the ordered cumulants". The following procedure and Lemma 2 remedies to this situation. 
By a recursive integration of the non-local equation (82) we get

$$
\begin{aligned}
\mathcal{M} & =1+\int_{0}^{t} \mathrm{~d} u_{1} \int_{0}^{u_{1}} \mathrm{~d} u_{1}^{\prime} G\left(u_{1}, u_{1}^{\prime}\right) \\
& +\int_{0}^{t} \mathrm{~d} u_{1} \int_{0}^{u_{1}} \mathrm{~d} u_{1}^{\prime} G\left(u_{1}, u_{1}^{\prime}\right) \otimes \int_{0}^{u_{1}^{\prime}} \mathrm{d} u_{2} \int_{0}^{u_{2}} \mathrm{~d} u_{2}^{\prime} G\left(u_{2}, u_{2}^{\prime}\right) \\
& +\int_{0}^{t} \mathrm{~d} u_{1} \int_{0}^{u_{1}} \mathrm{~d} u_{1}^{\prime} G\left(u_{1}, u_{1}^{\prime}\right) \otimes \int_{0}^{u_{1}^{\prime}} \mathrm{d} u_{2} \int_{0}^{u_{2}} \mathrm{~d} u_{2}^{\prime} G\left(u_{2}, u_{2}^{\prime}\right) \otimes \int_{0}^{u_{2}^{\prime}} \mathrm{d} u_{3} \int_{0}^{u_{3}} \mathrm{~d} u_{3}^{\prime} G\left(u_{3}, u_{3}^{\prime}\right)+\ldots \\
& :=\exp _{G}\left[\int_{0}^{t} \mathrm{~d} u \int_{0}^{u} \mathrm{~d} u^{\prime} G\left(u, u^{\prime}\right)\right]:=\exp _{G}[\mathcal{K}(t)]
\end{aligned}
$$

Thus, $M_{O}=G$. Assuming the expansion $G\left(u, u^{\prime}\right)=\sum_{1}^{\infty} G_{n}\left(u, u^{\prime}\right)$ and making the association

$$
\int_{0}^{t} \mathrm{~d} u \int_{0}^{u} \mathrm{~d} u^{\prime} G_{n}\left(u, u^{\prime}\right)=\mathcal{K}_{n}(t)
$$

we have:

$$
\int_{0}^{t} \mathrm{~d} u \int_{0}^{u} \mathrm{~d} u^{\prime} G_{1}\left(u, u^{\prime}\right)=\mathcal{K}_{1}(t)=\mathcal{M}_{1}(t)
$$

from which, with some arbitrariness, we can set,

$$
G_{1}\left(u, u^{\prime}\right)=\delta\left(u-u^{\prime}\right)\langle\Omega(u)\rangle
$$

and it follows that

$$
\begin{aligned}
& \int_{0}^{t} \mathrm{~d} u \int_{0}^{u} \mathrm{~d} u^{\prime} G_{2}\left(u, u^{\prime}\right)=\mathcal{K}_{2}(t) \\
& =\int_{0}^{t} \mathrm{~d} u_{1} \int_{0}^{u_{1}} \mathrm{~d} u_{2}\left\langle\Omega\left(u_{1}\right) \Omega\left(u_{2}\right)\right\rangle_{c} .
\end{aligned}
$$

Eq. (87) leads to the following identifications: $u=u_{1}$ and $u^{\prime}=u_{2}$, and

$$
G_{2}\left(u, u^{\prime}\right)=\left\langle\Omega(u) \Omega\left(u^{\prime}\right)\right\rangle_{c}
$$

Going ahead in this way, we see that for $n \geq 3$ there is ambiguity in the identification of the time $u^{\prime}$ of $G_{n}\left(u, u^{\prime}\right)$ with one of the times $u_{2}, u_{3}, \ldots, u_{n}$ of the $n$-cumulant density function. The ambiguity is made clear by deriving respect to the time $t$ both sides of Eq. (84), from which we get

$$
\int_{0}^{t} d u^{\prime} G_{n}\left(t, u^{\prime}\right)=\int_{0}^{t} \mathrm{~d} u_{2} \int_{0}^{u_{2}} \mathrm{~d} u_{3} \ldots \int_{0}^{u_{n-1}} \mathrm{~d} u_{n}\left\langle\Omega(t) \Omega\left(u_{2}\right) \Omega\left(u_{3}\right) \ldots \Omega\left(u_{n}\right)\right\rangle_{c} .
$$

From the above equation it is clear that the first time parameter $u$ of $G_{n}\left(u, u^{\prime}\right)$ is uniquely associated to the first one, $u_{1}$, of the generalized cumulant density function $\left.\Omega(t) \Omega\left(u_{2}\right) \Omega\left(u_{3}\right) \ldots \Omega\left(u_{n}\right)\right\rangle_{c}$. 
On the other hand, for $n>2$ we have $n-1$ different possibilities for the second time parameter $u^{\prime}: u^{\prime}=u_{2}, u^{\prime}=u_{3}, \ldots, u^{\prime}=u_{n}$ (see Appendix C). Let us make the choice $u^{\prime}=u_{n}$, thus (see Eq. (C4) of Appendix C):

$$
G_{n}\left(u, u^{\prime}\right)=\int_{u^{\prime}}^{u} \mathrm{~d} u_{2} \int_{u^{\prime}}^{u_{2}} \mathrm{~d} u_{3} \ldots \int_{u^{\prime}}^{u_{n-2}} \mathrm{~d} u_{n-1}\left\langle\Omega(u) \Omega\left(u_{2}\right) \Omega\left(u_{3}\right) \ldots \Omega\left(u_{n-1}\right) \Omega\left(u^{\prime}\right)\right\rangle_{c},
$$

or

$$
\begin{aligned}
\mathcal{K}_{n}(t) & =\int_{0}^{t} \mathrm{~d} u \int_{0}^{u} \mathrm{~d} u^{\prime} G_{n}\left(u, u^{\prime}\right) \\
& =\int_{0}^{t} \mathrm{~d} u \int_{0}^{u} \mathrm{~d} u_{2} \int_{0}^{u_{2}} \mathrm{~d} u_{3} \ldots \int_{0}^{u_{n-1}} \mathrm{~d} u^{\prime}\left\langle\Omega(u) \Omega\left(u_{2}\right) \Omega\left(u_{3}\right) \ldots \Omega\left(u^{\prime}\right)\right\rangle_{c}
\end{aligned}
$$

Exploiting Eq. (91) in the series of Eq. (83), we see that in this case the $M_{O}$ projection map must be defined in the following way: in any tensor product such as $(n, m \in \mathbb{N})$

$$
. . \otimes\left\langle\Omega\left(u_{i}\right) \Omega\left(u_{i+1}\right) \ldots \Omega\left(u_{i+n-1}\right)\right\rangle_{c} \otimes\left\langle\Omega\left(u_{j}\right) \Omega\left(u_{j+1}\right) \ldots \Omega\left(u_{i+m-1}\right)\right\rangle_{c} \otimes \ldots
$$

the $M_{O}$ map imposes a decreasing time ordering from left to right between the last parameter $u_{i+n-1}$ of any generalized cumulant density function and the first parameter $u_{j}$ of the next cumulant density function, namely, $u_{i+n-1} \geq u_{j}$. Because inside the cumulant density function the operators are ordered from left to right (we are considering the case where $M=O$ ), this $M_{O}$ map leads to a total time ordering (TTO) for the operators $\Omega(u)$ in any expression connecting generalized moments to generalized cumulants and vice-versa. Thus, Eq. (62) becomes: (again, we assume $u_{i}>u_{j}$ for $j>i$ ):

$$
\left\langle\Omega\left(u_{1}\right) \Omega\left(u_{2}\right) \ldots \Omega\left(u_{n}\right)\right\rangle=\left\{\begin{array}{l}
\sum_{\substack{\text { compositions } \\
\text { grouping } \\
\langle\Omega\rangle_{c}}}\left[\Omega\left(u_{1}\right) \Omega\left(u_{2}\right) \ldots \Omega\left(u_{n}\right)\right] \\
\left\langle\Omega\left(u_{1}\right)\left(1+\mathbb{P}_{c}\right) \Omega\left(u_{2}\right)\left(1+\mathbb{P}_{c}\right) \Omega\left(u_{3}\right) \ldots\left(1+\mathbb{P}_{c}\right) \Omega\left(u_{n}\right)\right\rangle
\end{array}\right.
$$

In Eq. (92a) the sum is over all the $2^{n-1}$ possible ordered compositions of $\Omega\left(u_{1}\right) \Omega\left(u_{2}\right) \ldots \Omega\left(u_{n}\right)$, with grouping made by using the average $\langle\ldots\rangle_{c}$. In Eq. (92b) the projection operator $\mathbb{P}_{c}$ breaks a cumulant average in the product of two averages: "... $\left.\mathbb{P}_{c} \ldots=\ldots\right\rangle_{c} \otimes\langle\ldots$ ". A generic term of the sum is given by the product of $p$ cumulant density functions:

$$
\begin{aligned}
& \left\langle\Omega\left(u_{1}\right) \ldots \Omega\left(u_{m_{1}}\right)\right\rangle_{c} \otimes\left\langle\Omega\left(u_{m_{1}+1}\right) \ldots \Omega\left(u_{m_{1}+m_{2}}\right)\right\rangle_{c} \otimes \ldots \\
& \ldots \otimes\left\langle\Omega\left(u_{m_{1}+m_{2}+\ldots+m_{p-1}+1}\right) \ldots \Omega\left(u_{m_{1}+m_{2}+\ldots+m_{p-1}+m_{p}}\right)\right\rangle_{c}
\end{aligned}
$$

such that $m_{1}+m_{2}+\ldots+m_{p-1}+m_{p}=n$ (i.e., the last time index must be equal to $n$ ). For fixed $p$ groups we have $\frac{(n-1) !}{(p-1) !(n-p) !}$ possible ways of grouping, of course, $\sum_{p=1}^{n} \frac{(n-1) !}{(p-1) !(n-p) !}=2^{n-1}$. 
The first four density moments in terms of cumulants can be written by using Eq. (92), or, more easily, we can take advantage of the partial ordered result of Eq. (79), and discard, in the same equation, all the terms that are non totally ordered:

$$
\begin{aligned}
\langle 1 \cdot 2\rangle & =\langle 1 \cdot 2\rangle_{c}+\langle 1\rangle \otimes\langle 2\rangle \\
\langle 1 \cdot 2 \cdot 3\rangle & =\langle 1 \cdot 2 \cdot 3\rangle_{c}+\langle 1\rangle \otimes\langle 2\rangle \otimes\langle 3\rangle \\
{[3 \text { terms } \Rightarrow 1 \text { term: }] } & +\langle 1 \cdot 2\rangle_{c} \otimes\langle 3\rangle+\langle 1\rangle \otimes\langle 2 \cdot 3\rangle_{c} \\
\langle 1 \cdot 2 \cdot 3 \cdot 4\rangle & =\langle 1 \cdot 2 \cdot 3 \cdot 4\rangle_{c}+\langle 1\rangle \otimes\langle 2\rangle \otimes\langle 3\rangle \otimes\langle 4\rangle \\
{[4 \text { terms } \Rightarrow 2 \text { terms: }] } & +\langle 1 \cdot 2 \cdot 3\rangle_{c} \otimes\langle 4\rangle+\langle 1\rangle \otimes\langle 2 \cdot 3 \cdot 4\rangle_{c} \\
{[3 \text { terms } \Rightarrow 1 \text { term: }] } & +\langle 1 \cdot 2\rangle_{c} \otimes\langle 3 \cdot 4\rangle_{c} \\
{[6 \text { terms } \Rightarrow 3 \text { terms: }] } & +\langle 1 \cdot 2\rangle_{c} \otimes\langle 3\rangle \otimes\langle 4\rangle \\
& +\langle 1\rangle \otimes\langle 2 \cdot 3\rangle_{c} \otimes\langle 4\rangle+\langle 1\rangle \otimes\langle 2\rangle \otimes\langle 3 \cdot 4\rangle_{c}
\end{aligned}
$$

The relation of Eq. (92) (or of Eq. (94)) is easily invertible and gives:

$$
\left\langle\Omega\left(u_{1}\right) \Omega\left(u_{2}\right) \ldots \Omega\left(u_{n}\right)\right\rangle_{c}=\left\langle\Omega\left(u_{1}\right)(1-\mathbb{P}) \Omega\left(u_{2}\right)(1-\mathbb{P}) \Omega\left(u_{3}\right) \ldots(1-\mathbb{P}) \Omega\left(u_{n}\right)\right\rangle
$$

in which the projection operator $\mathbb{P}$ is similar to $\mathbb{P}_{c}$ but acts to standard moment averages, instead of cumulant ones "...P... = ... $\langle\ldots$ ". As for Eq. (92), the r.h.s. of Eq. (95) is the sum of $2^{n-1}$ terms, each one corresponding to one of the possible different ways to make a partition of the ordered sequence of $n$ operators $\Omega\left(u_{i}\right), 0 \leq i \leq n$, in $p \leq n$ groups (the moments), such that the time ordering is fully preserved (namely $u_{i}>u_{j}$ for $j>i$ ) and suppling the partition with a factor $(-1)^{p+1}$. A generic term of the sum is given by

$$
\begin{aligned}
(-1)^{p+1}\left\langle\Omega\left(u_{1}\right) \ldots \Omega\left(u_{m_{1}}\right)\right\rangle\left\langle\Omega\left(u_{m_{1}+1}\right) \ldots \Omega\left(u_{m_{1}+m_{2}}\right)\right\rangle \ldots \\
\ldots\left\langle\Omega\left(u_{m_{1}+m_{2}+\ldots+m_{p-1}+1}\right) \ldots \Omega\left(u_{m_{1}+m_{2}+\ldots+m_{p-1}+m_{p}}\right)\right\rangle
\end{aligned}
$$

where $m_{1}+m_{2}+\ldots+m_{p-1}+m_{p}=n$. As we have reported at the beginning of this Section, we are now in the position to make a direct link between the cumulant approach and the Zwanzig 53,54 perturbation projection procedure: from Eq. (95), the $n$th cumulant generating component $\mathcal{K}_{n}=\int_{0}^{t} \mathrm{~d} u_{1} \int_{0}^{u_{1}} \mathrm{~d} u_{2} \ldots \int_{0}^{u_{n-1}} \mathrm{~d} u_{n}\left\langle\Omega\left(u_{1}\right) \ldots \Omega\left(u_{n}\right)\right\rangle_{c}$ corresponds to the $n$th order of the series expansion we get from the Zwanzig projection method. This fact, and the fundamental property for cumulants (Lemma 2), prove the connection between the Terwiel $^{23}$ cluster property for the "partial kernels" arising from the Zwanzig approach and the factorization property of the ordered cumulants. 
In the case where the stochastic process is stationary, the integral form of Eq. (82) becomes a convolution, thus it can easily Fourier-transformed. From the relation of Eq. (84), we have $\hat{G}(\nu)=\nu^{2} \hat{\mathcal{K}}(\nu)$. In general, the TTO cumulant approach can be useful for analysis performed in the frequency domain, sometimes the only possible way for anomalous diffusion processes.

Eq. (79) differs from Eq. (94) because the former considers also cross partitions of the numerals $1,2 \ldots, n$, while the latter only ordered (non-crossing) ones. Thus, starting from the reverse relation of Eq. (95), by using combinatorial (or graph) arguments, it is easy to obtain the procedure of Roerdnik ${ }^{47}$, which gives cumulants in terms of moments also for the previous example, where the moment generating function satisfies the local time equation of Eq. (75) :

(i) Write a sequence of $n$-dots.

(ii) Write 1 on the first dot, and any permutation of the numerals $2,3, \ldots ., n$ on the remaining dots.

(iii) Partition each of the $(n-1)$ ! permutations of numerals into subsequences by inserting TTO cumulant averages $\langle\ldots \ldots\rangle_{c}$ with the constraint that two successive numerals belong to the same subsequence if and only if the first one is smaller than the second.

(iv) For each partition consisting of $p$ subsequences supply a factor $(-1)^{p+1}$.

(v) Replace each numeral " $\imath$ " by " $\Omega\left(u_{i}\right)$ ".

Eqs. (95) and the related above procedure of Roerdnik are here obtained in a very general context, and can always be applied when the $M$ map is the time ordering corresponding to a $t$-ordered exponential and when the moment generating function satisfies an integral (or time-convolution) equation or a time local (or time-convolutionless) equation, respectively. For example, the expansion formulas of Eqs. (5.9)-(5.10) and Eqs. (5.17)-(5.18) of $\frac{55}{55}$ can be directly obtained by using Eqs. (95)) and the above Roerdnik's procedure, respectively.

\section{CONCLUSIONS}

In this work, we revisit the classical Kubo's approachㄴ.2 (referred as K62-63 in this paper) that introduces moment-operators and cumulant-operators. The Kubo's idea of extending 
to non-commuting quantities the concept of cumulants has been used by many researchers in the last fifty years as a tool for some systematic expansion of, for example, the Liouville equation of both classical systems $\underline{21-23,56}$ and spin systems (e.g. $\underline{10,11}$ ), or to separate the non extensive parts of the reduced density matrix for many body boson or fermion systems (e.g., $2-19 \underline{19}$ ). However, apart from the general idea, usually the results of these old works of Kubo have not been so exploited. A more case-dependent and analytical approach has been instead adopted. This is because, as it was pointed out, for example, by Fox ${ }^{6.7}$, in K62-63 there are some theoretical gaps/flaws and, unfortunately, at least questionable results, that have never been fully cured so far.

In extending to non-commuting quantities the concept of cumulants a way to generalize the definition of exponential of operators must be introduced, so as to recover the factorization property of the exponential of a sum. Here we give indications on how to do that.

The generalized moment generating function is usually identified by the "facts" of the specific problem (e.g., from physics) we are interested in. For example, in the case of fermions a natural $\tilde{M}$ map is the physical constraints that the creation operators stay at the left of the annihilation ones and that the average is made with a totally antisymmetric function. Because in this case we are interested in the reduced density matrices (RDM see IVD 1), this $\tilde{M}$ map naturally leads to Eq. (67) as the RDM-generating function.

Once the generalized moments have been defined, the generalized exponential function that define the cumulant generating function can be chosen in different ways, depending on the specific expansion we are interested in. The fundamental requirement is that any cumulant that mixes independent "processes" should be zero. A property which, for noncommuting cumulants, is closely linked to the factorization property of the exponentials of the sums. In this work we faced the problem of how to introduce the generalized exponential for cumulant operators, in such a way to automatically meet this strong requirement. With Lemma 2 and Lemma 3 we provide a solution to this problem, suitable for a wide class of practical cases.

Moreover, we also clarify that, in spite of what stated in K62-63, a general Meeron formula that gives generalized cumulants in terms of generalized moments cannot be given, but the reverse is easily obtained (see Lemma 4 and Eqs. (62)-(63)). It is noticeable that its formal expression does not depend on the specific definition of generalized moments, but only on the chosen definition of generalized cumulants (the $M_{O}$ map). 
Applications of the results of the present paper to different problems in physics are briefly illustrated, emphasizing how it is possible to recover, in an unique theoretical framework, many different results that have been obtained by using case-dependent analytical approaches.

Other applications to new problems are under work.

\section{Appendix A: A precise definition of "time ordering"}

Let us consider the $M$-projection map corresponding to the chronological ordering of operators, from right (smaller times) to left (larger times). Thus, using the notation $\{\ldots\}_{O}$ to indicate time ordering of the argument "...", for any two time dependent operators $\mathcal{A}(t)$ and $\mathcal{B}(t)$ we have

$$
\left\{\mathcal{A}\left(t_{1}\right) \otimes \mathcal{B}\left(t_{2}\right)\right\}_{O}:=\left\{\begin{array}{l}
\mathcal{A}\left(t_{1}\right) \otimes \mathcal{B}\left(t_{2}\right) \text { for } t_{1}>t_{2} \\
\frac{\mathcal{B}\left(t_{1}\right) \otimes \mathcal{A}\left(t_{1}\right)+\mathcal{A}\left(t_{1}\right) \otimes \mathcal{B}\left(t_{1}\right)}{2} \text { for } t_{2}=t_{1} \\
\mathcal{B}\left(t_{2}\right) \otimes \mathcal{A}\left(t_{1}\right) \text { for } t_{2}>t_{1} .
\end{array}\right.
$$

Now, we assume that $t_{1}>t_{2}$ and let us consider the sums $S_{\mathcal{A}}\left(t_{1}, t_{2}\right):=\mathcal{A}\left(t_{1}\right)+\mathcal{A}\left(t_{2}\right)$ and $S_{\mathcal{B}}\left(t_{1}, t_{2}\right):=\mathcal{B}\left(t_{1}\right)+\mathcal{B}\left(t_{2}\right)$. One would be naturally tempted to write:

$$
\left\{S_{\mathcal{A}}\left(t_{1}, t_{2}\right) \otimes S_{\mathcal{B}}\left(t_{1}, t_{2}\right)\right\}_{O}=\mathcal{A}\left(t_{1}\right) \otimes \mathcal{B}\left(t_{1}\right)+\mathcal{A}\left(t_{1}\right) \otimes \mathcal{B}\left(t_{2}\right)+\mathcal{B}\left(t_{1}\right) \otimes \mathcal{A}\left(t_{2}\right)+\mathcal{A}\left(t_{2}\right) \otimes \mathcal{B}\left(t_{2}\right) .
$$

The above definition of time order looks clear, but it present some pitfalls, in fact it invalidates the distributive property of the usual algebra associated to operators of interest (like differential operators). This is clearly seen with the following two examples. In the first one we have $\mathcal{A}(t)=\mathcal{B}(t)=t \mathcal{C}+\mathcal{D}$, in which the operators $\mathcal{C}$ and $\mathcal{D}$ does not depend on time. From the l.h.s. of Eq. (A2) we have

$$
\begin{aligned}
& \left\{\left(\left(t_{1} \mathcal{C}+\mathcal{D}\right)+\left(t_{2} \mathcal{C}+\mathcal{D}\right)\right) \otimes\left(\left(t_{1} \mathcal{C}+\mathcal{D}\right)+\left(t_{2} \mathcal{C}+\mathcal{D}\right)\right)\right\}_{O} \\
& \left.\left.=\left\{\left(\left(t_{1}+t_{2}\right) \mathcal{C}+2 \mathcal{D}\right)\right)\left(\left(t_{1}+t_{2}\right) \mathcal{C}+2 \mathcal{D}\right)\right)\right\}_{O} \\
& =\left(t_{1}+t_{2}\right)^{2} \mathcal{C}^{2}+4 \mathcal{D}^{2}+2\left(t_{1}+t_{2}\right)(\mathcal{C} \otimes \mathcal{D}+\mathcal{D} \otimes \mathcal{C})
\end{aligned}
$$

while from the r.h.s. of Eq. (A2) we have

$$
\left(t_{1}+t_{2}\right)^{2} \mathcal{C}^{2}+4 \mathcal{D}^{2}+\left(t_{1}+t_{2}\right)(\mathcal{C} \otimes \mathcal{D}+\mathcal{D} \otimes \mathcal{C})+2 t_{1}\left(\mathcal{C} \otimes \mathcal{D}+2 t_{2} \mathcal{D} \otimes \mathcal{C}\right)
$$


Eq. (A3) differs from ( $(\underline{A} 4)$ by the term $\left(t_{1}+t_{2}\right)(\mathcal{D} \otimes \mathcal{C}-\mathcal{C} \otimes \mathcal{D})$. In words, the difference is due to the fact that if we first make the sum $\mathcal{S}$ of the operators evaluated at different times $\left(\mathcal{A}\left(t_{1}\right)+\mathcal{A}\left(t_{2}\right)+..\right)$, as in Eq. (A3), we loose the detailed information of the value of the operators at the partial times $t_{1}, t_{2}, \ldots$, thus the $M$-projection map is not involved.

The second example is an explicit case of the first one, where $\mathcal{C}=t y \partial_{x}$ and $\mathcal{D}=\partial_{y}$, namely $\mathcal{A}(t)=t y \partial_{x}+\partial_{y}$. Moreover, we assume also that the sum $S_{\mathcal{A}}$ is extended to an infinite series of times: $t_{0}=0, t_{1}=t / N, t_{2}=2 t / N, . ., t_{N}=N t / N=t, N \rightarrow \infty$. Therefore we have

$$
S_{\mathcal{A}}(0, t):=\int_{0}^{t} \mathcal{A}(u) \mathrm{d} u=\int_{0}^{t}\left(u y \partial_{x}+\partial_{y}\right) \mathrm{d} u=\frac{t^{2}}{2} y \partial_{x}+t \partial_{y} .
$$

In the time ordered product $\left\{S_{\mathcal{A}}(t) S_{\mathcal{A}}(t)\right\}_{O}$, if we first solve the integral, then we have just the square of an operator evaluated at the final time $t$ and the $M$-projection map is not effective:

$$
\begin{aligned}
\left\{S_{\mathcal{A}}(0, t) \otimes S_{\mathcal{A}}(0, t)\right\}_{O} & =\left\{\int_{0}^{t} \mathcal{A}(u) \mathrm{d} u \otimes \int_{0}^{t} \mathcal{A}(u) \mathrm{d} u\right\}_{O} \\
& =\left(\frac{t^{2}}{2} y \partial_{x}+t \partial_{y}\right)^{2}=\frac{t^{4}}{4} y^{2} \partial_{x}^{2}+\frac{t^{3}}{2}\left(y \partial_{x} \partial_{y}+\partial_{y} y \partial_{x}\right)+t^{2} \partial_{y}^{2} .
\end{aligned}
$$

But if the integrals are made after the time ordering, we get:

$$
\begin{aligned}
\left\{S_{\mathcal{A}}(0, t) \otimes S_{\mathcal{A}}(0, t)\right\}_{O} & =\left\{\int_{0}^{t} \mathcal{A}(u) \mathrm{d} u \otimes \int_{0}^{t} \mathcal{A}(u) \mathrm{d} u\right\}_{O} \\
& =\int_{0}^{t} \mathrm{~d} u_{1} \int_{0}^{t} \mathrm{~d} u_{2}\left\{\left(u_{1} y \partial_{x}+\partial_{y}\right)\left(u_{2} y \partial_{x}+\partial_{y}\right)\right\}_{O} \\
& =2 \int_{0}^{t} \mathrm{~d} u_{1} \int_{0}^{u_{1}} \mathrm{~d} u_{2}\left(u_{1} y \partial_{x}+\partial_{y}\right)\left(u_{2} y \partial_{x}+\partial_{y}\right) \\
& =\frac{t^{4}}{4} y^{2} \partial_{x}^{2}+\frac{t^{3}}{3}\left(2 y \partial_{x} \partial_{y}+\partial_{y} y \partial_{x}\right)+t^{2} \partial_{y}^{2},
\end{aligned}
$$

that is different from Eq. (A5). The $t$-ordered exponential is affected by this problem because is a series of terms like $\left\{S_{\mathcal{A}}(0, t)^{n}\right\}_{O}$. On the other hand, if in the above example we state that the action of the time ordering map $\{\ldots\}_{O}$ is defined only on the special basis generated by the tensor products of $\mathcal{A}(u), u \in[0, t]$, then, when it is applied to the vector $S_{\mathcal{A}}(0, t)$, this last must be first decomposed in the special basis: $S_{\mathcal{A}}(0, t):=\int_{0}^{t} \mathcal{A}(u) \mathrm{d} u$. In this way we avoid any apparent paradox. This is the reason for which we have introduced the point (ii) in the Definition $\mathrm{A}$ of the $M$-projection map.

Remark O. It is trivial but important to notice that in the case where the times of integration (or the sums) are not overlapping, for example $\left\{S_{\mathcal{A}}\left(t_{1}, t\right) S_{\mathcal{A}}\left(0, t_{1}\right)\right\}_{O}$ with $0 \leq t_{1} \leq t$, we 
recover the distributive property of the tensor multiplication combined with the $M$-projection map. This is because in this case the non overlapping time intervals defines a partition of $M$-unconnetced sets on $\mathfrak{M}$ (see Definition $\mathbb{C}$ ).

\section{Appendix B: A demonstration of the Meeron formula}

Proof of Proposition [2. The demonstration is straightforward and well known for commuting stochastic processes, for which the $M$-projection is just the identity mapping. Here the sketch: exploiting the multinomial theorem, Eq. (55) becomes

$$
\sum_{n=0}^{\infty} \mathcal{M}_{n}=\sum_{m=0}^{\infty} \sum_{\text {Comp.of } m}\left\{\prod_{r=1}^{\infty} \frac{1}{s_{r} !}\left(\mathcal{K}_{r}\right)^{s_{r}}\right\}_{M_{O}}
$$

where the sum of compositions of $m$ means sum over any infinite set of non negative integers $s_{1}, s_{2}, .$. with the constraint $\sum_{r=1}^{\infty} s_{r}=m$. Because $m$ can assume any value, we can rearrange the r.h.s. of Eq. (B1) as a sum over partitions of $n$ defined by $\sum_{r=1}^{n} r s_{r}=n$, as in the statement of the theorem:

$$
\sum_{n=0}^{\infty} \mathcal{M}_{n}=\sum_{n=0}^{\infty} \sum_{\text {Part. of } n}\left\{\prod_{r=1}^{n} \frac{1}{s_{r} !}\left(\mathcal{K}_{r}\right)^{s_{r}}\right\}_{M_{O}}
$$

In the r.h.s. of the above equation, for any fixed $n$ we have sums of terms of order $\sum_{r=1}^{n} r s_{r}=$ $n$, thus, by equating terms of the same order of both sides of Eq. (B2) the demonstration end.

\section{Appendix C: How to switch the time integrations}

Here we demonstrate that the parameter $u^{\prime}$ of the 1.h.s. of Eq. (89) can be associated with anyone of the $n-1$ parameters $u_{2}, \ldots, u_{n}$ of the r.h.s. of the same equation.

Getting rid of the first integral in both the sides of Eq. (89), one could be tempted to define $u^{\prime}=u_{2}$, from which

$$
G_{n}\left(u, u^{\prime}\right)=\int_{0}^{u^{\prime}} \mathrm{d} u^{\prime} \ldots \int_{0}^{u_{n-1}} \mathrm{~d} u_{n}\left\langle\Omega(u) \Omega\left(u^{\prime}\right) \Omega\left(u_{3}\right) \ldots \Omega\left(u_{n}\right)\right\rangle_{c} .
$$

With this choice the time ordering associated with the generalized exponential defined by the series (83), with the identification written in Eq. (844), would involve the first time $u=u_{1}$ and to the second time $u^{\prime}=u_{2}$ of the density cumulants $\left\langle\Omega\left(u_{1}\right) \Omega\left(u_{2}\right) . . \Omega\left(u_{n}\right)\right\rangle_{c}$. However, 
before to get rid of the first integral, in the r.h.s. of Eq. (89) we can change the order of integration between any couple integrals, let us say that involving $u_{i}$ and $u_{i-1}$ :

$$
\int_{0}^{u_{i-2}} \mathrm{~d} u_{i-1} \int_{0}^{u_{i-1}} \mathrm{~d} u_{i} \ldots=\int_{0}^{u_{i-2}} \mathrm{~d} u_{i} \int_{u_{i}}^{u_{i-2}} \mathrm{~d} u_{i-1} \ldots
$$

and, subsequently repeating this integral switch, we get (we have already set $u_{1}=u$ )

$$
\begin{aligned}
& \int_{o}^{u} d u^{\prime} G_{n}\left(u, u^{\prime}\right)=\int_{0}^{u} \mathrm{~d} u_{i} \int_{u_{i}}^{u} \mathrm{~d} u_{2} \int_{u_{i}}^{u_{2}} \mathrm{~d} u_{3} \ldots \int_{u_{i}}^{u_{i-2}} \mathrm{~d} u_{i-1} \int_{0}^{u_{i}} \mathrm{~d} u_{i+1} \ldots \\
& \ldots \int_{0}^{u_{n-2}} \mathrm{~d} u_{n-1} \int_{0}^{u_{n-1}} \mathrm{~d} u_{n}\left\langle\Omega(u) \Omega\left(u_{2}\right) \Omega\left(u_{3}\right) \ldots \Omega\left(u_{i}\right) \ldots \Omega\left(u_{n}\right)\right\rangle_{c} .
\end{aligned}
$$

Now, getting rid the first integral in both sides of this equation, we are lead to set $u^{\prime}=u_{i}$, from which

$$
\begin{aligned}
& G_{n}\left(u, u^{\prime}\right)=\int_{u^{\prime}}^{u} \mathrm{~d} u_{2} \int_{u^{\prime}}^{u_{2}} \mathrm{~d} u_{3} \ldots \int_{u^{\prime}}^{u_{i-2}} \mathrm{~d} u_{i-1} \int_{0}^{u^{\prime}} \mathrm{d} u_{i+1} \ldots \\
& \ldots \int_{0}^{u_{n-2}} \mathrm{~d} u_{n-1} \int_{0}^{u_{n-1}} \mathrm{~d} u_{n}\left\langle\Omega(u) \Omega\left(u_{2}\right) \Omega\left(u_{3}\right) \ldots \Omega\left(u^{\prime}\right) \ldots \Omega\left(u_{n}\right)\right\rangle_{c},
\end{aligned}
$$

\section{REFERENCES}

${ }^{1}$ R. Kubo, "Generalized cumulant method," Journal of the Physical Society of Japan 17, 1100-1120 (1962),

https://doi.org/10.1143/JPSJ.17.1100.

${ }^{2}$ R. Kubo, "Stochastic liouville equations," Journal of Mathematical Physics 4, 174-183 (1963), https://doi.org/10.1063/1.1703941.

${ }^{3} \mathrm{P}$. Laplace, Mémoire sur les approximations des formules qui sont fonctions de très grands nombres et sur leur application aux probabilité, edited by M. A. S. Paris.

${ }^{4}$ P. Laplace, Théorie analytique des probabilités, 2nd ed., edited by Coucir.

${ }^{5}$ P. Laplace, Théorie analytique des probabilités, 3rd ed., edited by Coucir.

${ }^{6}$ R. F. Fox, "Critique of the generalized cumulant expansion method," Journal of Mathematical Physics 17, 1148-1153 (1976), https://doi.org/10.1063/1.523041.

${ }^{7}$ R. F. Fox, "Time ordered operator cumulants: Statistical independence and noncommutativity," Journal of Mathematical Physics 20, 2467-2470 (1979), https://doi.org/10.1063/1.524055. 
${ }^{8}$ A. Nica and R. Speicher, "Commutators of free random variables," Duke Math. J. 92, 553-592 (1998).

${ }^{9}$ F. Lehner, "Free cumulants and enumeration of connected partitions," European Journal of Combinatorics 23, 1025 - 1031 (2002).

${ }^{10}$ J. H. Freed, "Generalized cumulant expansions and spinâĂ̌rrelaxation theory," The Journal of Chemical Physics 49, 376-391 (1968), https://doi.org/10.1063/1.1669833

${ }^{11}$ B. Yoon, J. M. Deutch, and J. H. Freed, "A comparison of generalized cumulant and projection operator methods in spinâĂ̌relaxation theory," The Journal of Chemical Physics 62, 4687-4696 (1975), https://doi.org/10.1063/1.430417.

${ }^{12}$ J. T. Skolnik and D. A. Mazziotti, "Cumulant reduced density matrices as measures of statistical dependence and entanglement between electronic quantum domains with application to photosynthetic light harvesting," Phys. Rev. A 88, 032517 (2013).

${ }^{13} \mathrm{P}$. Ziesche, "Cumulant expansions of reduced densities, reduced density matrices, and green's functions," in Many-Electron Densities and Reduced Density Matrices, Mathematical and Computational Chemistry, edited by J. Cioslowski (Springer US, 2000) Chap. 3, pp. XIV, 301, 1st ed., 978-1-4615-4211-7.

${ }^{14}$ D. A. Mazziotti, "Approximate solution for electron correlation through the use of schwinger probes," Chemical Physics Letters 289, 419 - 427 (1998).

${ }^{15}$ D. A. Mazziotti, "3,5-contracted schrÃúdinger equation: Determining quantum energies and reduced density matrices without wave functions," International Journal of Quantum Chemistry 70, 557-570 (1998).

${ }^{16}$ T. JuhÃasz and D. A. Mazziotti, "The cumulant two-particle reduced density matrix as a measure of electron correlation and entanglement," The Journal of Chemical Physics 125, 174105 (2006), https://doi.org/10.1063/1.2378768.

${ }^{17}$ Y. Pavlyukh and J. Berakdar, "Accessing electronic correlations by half-cycle pulses and time-resolved spectroscopy," Phys. Rev. A 90, 053417 (2014).

${ }^{18}$ E. Ramos-Cordoba, P. Salvador, M. Piris, and E. Matito, "Two new constraints for the cumulant matrix," The Journal of Chemical Physics 141, 234101 (2014), https://doi.org/10.1063/1.4903449.

${ }^{19} \mathrm{~A}$. Raeber and D. A. Mazziotti, "Large eigenvalue of the cumulant part of the 
two-electron reduced density matrix as a measure of off-diagonal long-range order," Phys. Rev. A 92, 052502 (2015).

${ }^{20}$ For historical reason we shall use the definition of q-numbers as objects of a non commutative algebra, as opposed to c-numbers that are objects of a commutative algebra. The operators considered in the present work are generally q-numbers.

${ }^{21}$ N. V. Kampen, "A cumulant expansion for stochastic linear differential equations. i," Physica 74, 215 - 238 (1974).

${ }^{22}$ N. V. Kampen, "A cumulant expansion for stochastic linear differential equations. ii," Physica 74, 239 - 247 (1974).

${ }^{23}$ R. Terwiel, "Projection operator method applied to stochastic linear differential equations," Physica 74, 248 - 265 (1974).

${ }^{24}$ M. Casula, A. Rubtsov, and S. Biermann, "Dynamical screening effects in correlated materials: Plasmon satellites and spectral weight transfers from a green's function ansatz to extended dynamical mean field theory," Phys. Rev. B 85, 035115 (2012).

${ }^{25}$ L. Hedin, "Effects of recoil on shake-up spectra in metals," Physica Scripta 21, 477-480 (1980).

${ }^{26}$ F. Aryasetiawan, L. Hedin, and K. Karlsson, "Multiple plasmon satellites in na and al spectral functions from ab initio cumulant expansion," Phys. Rev. Lett. 77, 2268-2271 (1996).

${ }^{27}$ M. Guzzo, G. Lani, F. Sottile, P. Romaniello, M. Gatti, J. J. Kas, J. J. Rehr, M. G. Silly, F. Sirotti, and L. Reining, "Valence electron photoemission spectrum of semiconductors: Ab initio description of multiple satellites," Phys. Rev. Lett. 107, 166401 (2011).

${ }^{28}$ E. Müller-Hartmann, T. V. Ramakrishnan, and G. Toulouse, "Localized dynamic perturbations in metals," Phys. Rev. B 3, 1102-1119 (1971).

${ }^{29}$ J. J. Kas, J. J. Rehr, and L. Reining, "Cumulant expansion of the retarded one-electron green function," Phys. Rev. B 90, 085112 (2014).

${ }^{30}$ G. D. Mahan, Many-particle physics, 3e, edited by S. I. P. Limited, Physics of solids and liquids (Kluwer Academic/Plenum Publishers, 2008) p. 788.

${ }^{31}$ D. Mukherjee, "A coupled cluster approach to the electron correlation problem using a correlated reference state," in Recent Progress in Many-Body Theories: Volume 4, edited by E. Schachinger, H. Mitter, and H. Sormann (Springer US, Boston, MA, 1995) pp. 127-133.

${ }^{32}$ M. Hanauer and A. KÃúhn, "Meaning and magnitude of the reduced density matrix cu- 
mulants," Chemical Physics 401, 50-61 (2012), recent advances in electron correlation methods and applications.

${ }^{33}$ H. Honmi, Y. Hashizume, T. Nakajima, and S. Okamura, "Microscopic study on magnetocaloric and electrocaloric effects near the critical point," Physica A: Statistical Mechanics and its Applications 433, 126 - 135 (2015).

${ }^{34}$ M. Suzuki, "A semi-phenomenological theory of the second order phase transitions in spin systems. i," Journal of the Physical Society of Japan 22, 756-761 (1967), https://journals.jps.jp/doi/10.1143/JPSJ.22.757.

${ }^{35}$ M. Suzuki, "Fluctuation and Relaxation in Stochastic Systems," $\quad$ Progress of Theoretical Physics Supplement 69, 160-173 (1980), http://oup.prod.sis.lan/ptps/article-pdf/doi/10.1143/PTP.69.160/5349369/69-160.pdf.

${ }^{36}$ Y. V. Pereverzev, A. Pereverzev, and E. Prezhdo, "Smoluchowski equation in cumulant approximation," Journal of the Physical Society of Japan 82, 024001 (2013), https://doi.org/10.7566/JPSJ.82.024001.

${ }^{37}$ D. J. Schneider and J. H. Freed, "Spin relaxation and motional dynamics," in Advances in Chemical Physics (John Wiley\& Sons, Inc., 2007) pp. 387-527.

${ }^{38} \mathrm{M}$. Tokuyama, "On the theory of fluctuations around non-equilibrium steady states: A generalized time-convolutionless projector formalism," Physica A: Statistical Mechanics and its Applications 102, 399 - 430 (1980).

${ }^{39}$ M. Tokuyama, "Statistical-dynamical theory of nonlinear stochastic processes: Ii. time-convolutionless projector method in nonequilibrium open systems," Physica A: Statistical Mechanics and its Applications 109, 128 - 160 (1981).

${ }^{40}$ P. Talkner, E. Lutz, and P. Hänggi, "Fluctuation theorems: Work is not an observable," Phys. Rev. E 75, 050102 (2007).

${ }^{41}$ S. Bachmann, G. M. Graf, and G. B. Lesovik, "Time ordering and counting statistics," Journal of Statistical Physics 138, 333-350 (2010).

${ }^{42}$ As done by $\mathrm{Kubo}^{1}$, we could consider the case where the integral, in the r.h.s. of Eq- (39) is substituted with a sum over the components of a $N$ dimensional vector of stochastic operators; however, for the sake of simplicity, we shall consider only the case of stochastic processes depending on a continuous parameter, e.g. the time. Adapting the results to the case of discrete, finite or infinite sets of stochastic operators is straightforward.

${ }^{43}$ E. Meeron, "Series expansion of distribution functions in multicompo- 
nent fluid systems," The Journal of Chemical Physics 27, 1238-1246 (1957), https://doi.org/10.1063/1.1743985.

${ }^{44} \mathrm{~L} . \quad$ A. Apresyan, "Cumulant analysis of stochastic linear operators," Radiophysics and Quantum Electronics 21, 493-500 (1978).

${ }^{45}$ R. F. Fox, "A generalized theory of multiplicative stochastic processes using cumulant techniques," Journal of Mathematical Physics 16, 289-297 (1975), https://aip.scitation.org/doi/pdf/10.1063/1.522540.

${ }^{46}$ O. Arizmendi, T. Hasebe, F. Lehner, and C. Vargas, "Relations between cumulants in noncommutative probability," Advances in Mathematics 282, 56 - 92 (2015).

${ }^{47} \mathrm{~J}$. Roerdink, "Inhomogeneous linear random differential equations with mutual correlations between multiplicative, additive and initial-value terms," Physica A: Statistical Mechanics and its Applications 109, 23 - 57 (1981).

${ }^{48} \mathrm{~W}$. Kutzelnigg and D. Mukherjee, "Cumulant expansion of the reduced density matrices," The Journal of Chemical Physics 110, 2800-2809 (1999), https://doi.org/10.1063/1.478189.

${ }^{49}$ D. A. Mazziotti, ed., Reduced-Density-Matrix Mechanics: With Application to Many-Electron Atoms anc ADVANCES IN CHEMICAL PHYSICS No. 134 (John Wiley \& Sons, Inc., 2007).

${ }^{50}$ P.-O. Löwdin, "Quantum theory of many-particle systems. i. physical interpretations by means of density matrices, natural spin-orbitals, and convergence problems in the method of configurational interaction," Phys. Rev. 97, 1474-1489 (1955).

${ }^{51}$ M. Bianucci, "Large scale emerging properties from non Hamiltonian complex systems," Entropy 19 (2017), 10.3390/e19070302

${ }^{52} \mathrm{M}$. Bianucci, "On the correspondence between a large class of dynamical systems and stochastic processes described by the generalized Fokker Planck equation with state-dependent diffusion and drift coefficients," Journal of Statistical Mechanics: Theory and Experiment 2015, P05016 (2015).

${ }^{53} \mathrm{P}$. Grigolini and F. Marchesoni, "Basic description of the rules leading to the adiabatic elimination of fast variables," in Memory Function Approaches to Stochastich Problems in Condensed Matter, Advances in Chemical Physics, Vol. LXII, edited by M. W. Evans, P. Grigolini, and G. P. Parravicini (An Interscience Publication, John Wiley \& Sons, New York, 1985) Chap. II, p. 556.

${ }^{54}$ R. Zwanzig, ed., Nonequilibrium Statistical Mechanics (Oxford University Press, 2001). 
${ }^{55}$ C. Uchiyama and F. Shibata, "Unified projection operator formalism in nonequilibrium statistical mechanics," Phys. Rev. E 60, 2636-2650 (1999).

${ }^{56}$ R. F. Fox, "Application of cumulant techniques to multiplicative stochastic processes," Journal of Mathematical Physics 15, 1479-1483 (1974), https://doi.org/10.1063/1.1666835. 